\title{
Long-term optical spectrophotometric monitoring of comet C/1995 O1 (Hale-Bopp) ${ }^{\star}$
}

\author{
H. Rauer ${ }^{1}$, J. Helbert ${ }^{1}$, C. Arpigny ${ }^{2}$, J. Benkhoff ${ }^{1}$, D. Bockelée-Morvan ${ }^{3}$, H. Boehnhardt ${ }^{4}$, F. Colas ${ }^{5}$, J. Crovisier ${ }^{3}$, \\ O. Hainaut ${ }^{4}$, L. Jorda ${ }^{6}$, M. Kueppers ${ }^{7}$, J. Manfroid ${ }^{2}$, and N. Thomas ${ }^{8}$
}

1 DLR, Institute of Space Sensor Technology and Planetary Exploration, Rutherfordstr. 2, 12489 Berlin, Germany

2 Institut d'Astrophysique et Géophysique, Sart Tilman B5c, 4000 Liège, Belgium

${ }^{3}$ Observatoire de Paris-Meudon, 5 place Jules Janssen, 92195 Meudon, France

4 European Southern Observatory, Alonso de Cordova 3107, Santiago de Chile

5 Bureau des Longitudes, 77 avenue Denfert Rochereau, 75014 Paris, France

${ }^{6}$ Laboratoire d'Astrophysique de Marseille, Site Pereisc, 13376 Marseille Cedex 12, France

7 Physikalisches Institut, Univ. Bern, Sidlerstrasse 5, 3012 Bern, Switzerland

8 MPI für Aeronomie, Max-Planck-Str. 2, 37191 Katlenburg-Lindau, Germany

\begin{abstract}
We observed comet C/1995 O1 (Hale-Bopp) at 4.6-2.9 AU pre-perihelion and 2.8-12.8 AU post-perihelion with optical long-slit spectroscopy. Emission bands of $\mathrm{CN}, \mathrm{C}_{3}, \mathrm{C}_{2}$ and $\mathrm{NH}_{2}$ have been covered. Emission of $\mathrm{C}_{3}$ was detected up to 7.0 AU, and CN could be followed up to $9.8 \mathrm{AU}$ post-perihelion. Spatial column density profiles of the radicals have been used to derive effective parent Haser scale lengths for heliocentric distances beyond 3 AU. Production rates were derived based on these Haser scale lengths. The observations of $\mathrm{CN}$ are in agreement with $\mathrm{HCN}$ as the major parent molecule of this radical at large distances from the Sun (i.e. beyond $\sim 3 \mathrm{AU}$ ). We compare the measured $\mathrm{CN}$ production rate to sublimation rates of $\mathrm{HCN}$ from a simple nucleus sublimation model. The variation of $\mathrm{CN}$ production rates with changing heliocentric distance gives no indication for sublimation from the interior and is consistent with very little thermal lag of the nucleus.
\end{abstract}

Key words. comets: general - comets: individual: C/1995 O1 (Hale-Bopp) - solar system: general

\section{Introduction}

Soon after the discovery of comet Hale-Bopp (C/1995 O1) in autumn 1995 it became clear that this object would be among the exceptionally bright comets. To take advantage of this opportunity, a monitoring program was initiated, starting in spring 1996 (Rauer et al. 1997). The monitoring was performed at optical telescopes, taking long-slit spectra and images through broad-band filters. Most of the observations were obtained at the European Southern Observatory (ESO), Chile, using several telescopes ranging from $1.5 \mathrm{~m}$ class to the VLT (Very Large Telescope), plus some observations at the Observatoire de Haute-Provence (OHP), France. The observations continued for 5 years in total and ended in January 2001. They cover a range of heliocentric distances of 4.6-2.9 AU prior to perihelion, and 2.8-12.8 AU post-perihelion. Emission bands of the $\mathrm{CN}, \mathrm{C}_{3}, \mathrm{C}_{2}$ and $\mathrm{NH}_{2}$ radicals have been followed in this observing campaign.

Send offprint requests to: $\mathrm{H}$. Rauer, e-mail: heike.rauer@dlr.de

* Based on observations collected at the European Southern Observatory, Chile, and the Observatoire de Haute Provence, France.
Several other monitoring campaigns of comet Hale-Bopp have also been performed over the past years. The dust coma has been imaged in broad-band filters from ESO telescopes (e.g.: Boehnhardt et al. 1999). Optical photometric monitoring performed by Schleicher et al. (1997) studied OH, NH, $\mathrm{C}_{3}$ and $\mathrm{C}_{2}$ radicals. Furthermore, long-term observations of comet Hale-Bopp at radio and infrared wavelengths (Biver et al. 1999; Dello Russo et al. 2001) allowed us to follow the activity of many of the parent molecules sublimating from the nucleus.

A main subject of cometary science is to understand the composition and structure of the nucleus. The variation of production rates of the icy cometary constituents with changing heliocentric distance depends critically on the details of the sublimation processes. Unfortunately, the parameters determining cometary ice sublimation are only poorly known. The chemical composition, but also the physical parameters of the nucleus influence the activity. Observations of gas production rates of ices with different volatility can, however, provide constraints to models of the sublimation process. Observations over a wide range of heliocentric distances are essential for such comparison. Therefore, direct observations of 
Table 1. Instruments and telescopes used, their field of view (FOV), slit length for spectroscopy and spatial scale.

\begin{tabular}{|c|c|c|c|c|c|}
\hline "Observatory & Telescope & Instrument & $\begin{array}{c}\text { FOV } \\
\text { [arcmin] }\end{array}$ & $\begin{array}{l}\text { slit length } \\
\text { [arcmin] }\end{array}$ & $\begin{array}{c}\text { scale } \\
{[\operatorname{arcsec} / \text { pixel }]}\end{array}$ \\
\hline \multirow[t]{5}{*}{ ESO } & Danish $1.54 \mathrm{~m}$ & DFOSC & $13.7 \times 13.7$ & 13.7 & 0.39 \\
\hline & ESO $1.52 \mathrm{~m}$ & Boller\&Chivens* & & 4.5 & 0.82 \\
\hline & $2.2 \mathrm{~m}$ & EFOSC2 & $5.7 \times 5.7$ & 5.7 & 0.16 \\
\hline & $3.6 \mathrm{~m}$ & EFOSC2 & $5.7 \times 5.7$ & 5.7 & 0.16 \\
\hline & VLT (UT1) & FORS1 & $6.8 \times 6.8$ & 6.8 & 0.20 \\
\hline OHP & $1.93 \mathrm{~m}$ & CARELEC ${ }^{*}$ & & 5.5 & 1.10 \\
\hline
\end{tabular}

${ }^{*}$ No imaging capability.

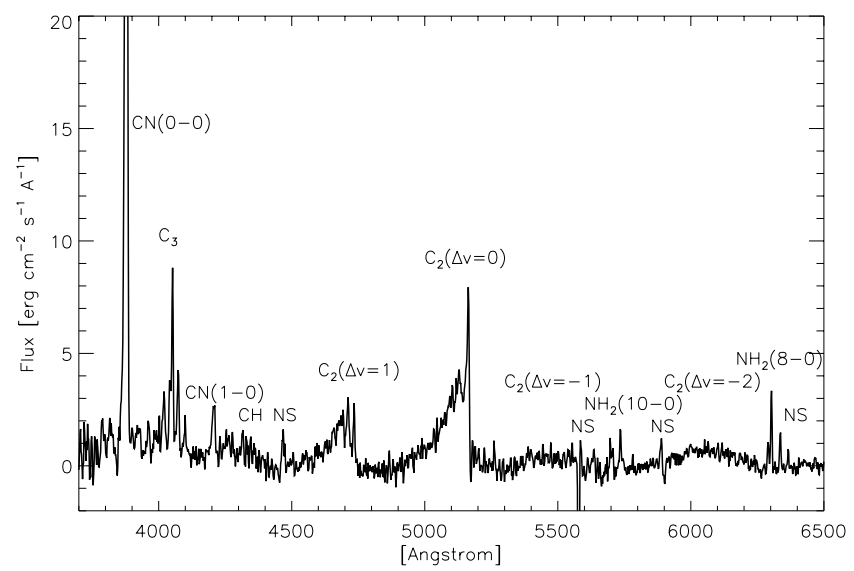

Fig. 1. Cut through a spectrum at $80000 \mathrm{~km}$ projected nucleocentric distance sunward. The spectrum was obtained on December 19, 1997 $\left(r_{\mathrm{h}}=3.8 \mathrm{AU}, 1200 \mathrm{~s}\right.$ exposure time). The underlying continuum level caused by solar light scattered on dust particles has been subtracted. The main gas emission bands are marked. Residuals of night sky emissions are indicated (NS)

parent molecules and sensitive optical observations of daughter radicals, which extend the range of distances covered, complement each other.

The long-slit column density profiles obtained in this study provide information on the spatial distribution of the radicals in the coma. In this paper, we present the observations, derive effective Haser parent scale lengths at distances beyond $3 \mathrm{AU}$, and study the evolution of gas production rates with heliocentric distance. The $B-X=(0-0)$ emission band of the $\mathrm{CN}$ radical at $3875 \AA$ is among the strongest emissions of gaseous species in comets. It is one of the few emissions which can be used to trace and characterize gas activity in weak and in particularly distant comets, where most other emissions of coma species are too weak to be observed. It is shown that $\mathrm{CN}$ production rates can be used as a measure of the production rate of its parent, $\mathrm{HCN}$, at the heliocentric distances covered here. The gas production rate evolution is compared to a simple $\mathrm{HCN}$ sublimation model, providing constraints to sublimation processes in the cometary nucleus.

\section{Observations and data reduction}

The observations of comet Hale-Bopp cover a period from April 1996 to October $1996\left(r_{\mathrm{h}}=4.6-2.9 \mathrm{AU}\right)$ prior to perihelion, and September 1997 to January $2001\left(r_{\mathrm{h}}=2.8-\right.$ 12.8 AU) post-perihelion. Most of the observations were performed at the European Southern Observatory (ESO), Chile. In May and September 1996, observations were made at the $1.93 \mathrm{~m}$ telescope of the Observatoire de Haute-Provence (OHP), France. The telescopes and instruments used are listed in Table 1. Medium resolution spectroscopy was performed. With the DFOSC, EFOSC2 and FORS1 instruments spectra were combined with imaging through broad-band filters $(R$ and $B)$ to image the two-dimensional coma dust distribution and record the position of the slit with respect to coma structures. A detailed overview of the observing circumstances is given in Table 2 .

In most observations, the slit has been aligned along the projected sun-comet line with the nucleus (brightest point) near the slit center. Occasionally, spectra have been taken at other position angles, or the nucleus position was displaced along the slit to cover a wider range along the sunward or tailward direction.

Bias frames, flatfields and wavelengths calibration exposures were taken and used for basic data reduction. The gas and dust coma of comet Hale-Bopp extended in most cases farther than the projected nucleocentric radius covered by the field of view (FOV). Therefore, separate spectra of the sky were taken, close in time to the comet exposures, several arcmin (up to 30 arcmin) offset from the nucleus position at the same elevation. The sky background in these spectra was averaged along the slit to increase the $S / N$-ratio and scaled to match the exposure time of the comet spectra. When comet Hale-Bopp was farther than about 9 AU from the Sun the coma was small compared to the FOV, and the sky background was obtained directly from the edges of the FOV of the comet spectra.

At the $1.5 \mathrm{~m}$ Danish telescope, equipped with DFOSC, stray radiation appeared in the blue wavelengths range (at less than about $4500 \AA$ ). This straylight pattern could not be completely removed during data reduction because of its variability due to flexure of the instrument. It, therefore, leads to an additional error, in particular near the position of the $\mathrm{C}_{3}$ emission band. Data of $\mathrm{C}_{3}$ emissions obtained at the $1.5 \mathrm{~m}$ Danish telescope have therefore not been used to derive parent Haser scale lengths in our further analysis.

Spectrophotometric standard stars have been observed every night for flux calibration (unless indicated otherwise in Table 2). Some nights were not photometric (in particular in mid-1998 the weather conditions were bad due to the El Niño 
Table 2. Overview of the observations. $r_{\mathrm{h}}$ and $\Delta$ denote the heliocentric and geocentric distances, respectively. $\beta$ provides the phase angle. Column 7 gives a list of the radicals detected at the respective heliocentric distance. Column 8 shows the wavelengths scale per pixel, and Col. 9 the slit width used.

\begin{tabular}{|c|c|c|c|c|c|c|c|c|c|}
\hline Date & $\begin{array}{r}r_{\mathrm{h}} \\
{[\mathrm{AU}]}\end{array}$ & $\begin{array}{r}\Delta \\
{[\mathrm{AU}]}\end{array}$ & $\begin{array}{r}\beta \beta \\
{\left[{ }^{\circ}\right]}\end{array}$ & Telescope & Instrument & Molecules & $\begin{array}{c}\Delta \lambda \\
{[\AA / / \text { pixel }]}\end{array}$ & $\begin{array}{l}\text { Slit } \\
{\left[{ }^{\prime \prime}\right]}\end{array}$ & Comments \\
\hline \multicolumn{10}{|c|}{ pre-perihelion } \\
\hline 23.-27.04.1996 & 4.6 & 4.3 & 12.4 & Danish $1.5 \mathrm{~m}$ & DFOSC & $\begin{array}{l}\mathrm{CN}, \mathrm{C}_{2}, \\
\mathrm{C}_{3}, \mathrm{NH}_{2}\end{array}$ & 3.0 & 2.0 & \\
\hline 28.-31.05.1996 & 4.3 & 3.5 & 9.6 & $1.93 \mathrm{~m}$ & CARELEC & $\mathrm{NH}_{2}$ & 1.8 & 2.2 & non-photom. \\
\hline 17.-26.06.1996 & 4.0 & 3.05 & 3.9 & Danish $1.5 \mathrm{~m}$ & DFOSC & $\begin{array}{l}\mathrm{CN}, \mathrm{C}_{2}, \\
\mathrm{C}_{3}, \mathrm{NH}_{2}\end{array}$ & 3.0 & 2.0 & \\
\hline 17.-24.08.1996 & 3.4 & 2.8 & 15.2 & Danish $1.5 \mathrm{~m}$ & DFOSC & $\begin{array}{l}\mathrm{CN}, \mathrm{C}_{2}, \\
\mathrm{C}_{3}, \mathrm{NH}_{2}\end{array}$ & 3.0 & $\begin{array}{l}1.0 \\
2.5\end{array}$ & \\
\hline 10.-16.09.1996 & 3.1 & 2.9 & 18.9 & $1.93 \mathrm{~m}$ & CARELEC & $\begin{array}{l}\mathrm{CN}, \mathrm{C}_{2}, \\
\mathrm{NH}_{2}\end{array}$ & 1.8 & 2.2 & \\
\hline 10.-16.09.1996 & 3.1 & 2.9 & 18.9 & $2.2 \mathrm{~m}$ & EFOSC2 & $\begin{array}{l}\mathrm{CN}, \mathrm{C}_{2}, \\
\mathrm{C}_{3}\end{array}$ & 1.5 & 2.0 & no calib. \\
\hline 1.-4.10.1996 & 2.9 & 3.0 & 19.5 & Danish $1.5 \mathrm{~m}$ & DFOSC & $\begin{array}{l}\mathrm{CN}, \mathrm{C}_{2}, \\
\mathrm{C}_{3}, \mathrm{NH}_{2}\end{array}$ & 3.0 & 2.5 & \\
\hline \multicolumn{10}{|c|}{ post-perihelion } \\
\hline $20 . / 21.09 .1997$ & 2.8 & 3.1 & 18.9 & $2.2 \mathrm{~m}$ & EFOSC2 & $\begin{array}{l}\mathrm{CN}, \mathrm{C}_{2}, \\
\mathrm{C}_{3}\end{array}$ & 1.5 & 2.0 & no calib. \\
\hline 29.9.-3.10.1997 & 2.9 & 3.1 & 18.8 & $2.2 \mathrm{~m}$ & EFOSC2 & $\begin{array}{l}\mathrm{CN}, \mathrm{C}_{2}, \\
\mathrm{NH}_{2}\end{array}$ & 2.0 & 1.5 & \\
\hline 23./24.11.1997 & 3.5 & 3.4 & 16.2 & ESO $1.5 \mathrm{~m}$ & $\mathrm{~B} \& \mathrm{C}$ & $\begin{array}{l}\mathrm{CN}, \mathrm{C}_{2}, \\
\mathrm{C}_{3}, \mathrm{NH}_{2}\end{array}$ & 1.9 & 2.4 & non-photom. \\
\hline 6.-9.12.1997 & 3.6 & 3.5 & 15.6 & Danish $1.5 \mathrm{~m}$ & DFOSC & $\begin{array}{l}\mathrm{CN}, \mathrm{C}_{2}, \\
\mathrm{C}_{3}, \mathrm{NH}_{2}\end{array}$ & 3.0 & 1.5 & \\
\hline 19./20.12.1997 & 3.8 & 3.6 & 15.0 & ESO $1.5 \mathrm{~m}$ & $\mathrm{~B} \& \mathrm{C}$ & $\begin{array}{l}\mathrm{CN}, \mathrm{C}_{2}, \\
\mathrm{C}_{3}, \mathrm{NH}_{2}\end{array}$ & 1.9 & 2.4 & \\
\hline 20./21.01.1998 & 4.1 & 4.0 & 13.8 & ESO $1.5 \mathrm{~m}$ & $\mathrm{~B} \& \mathrm{C}$ & $\begin{array}{l}\mathrm{CN}, \mathrm{C}_{2}, \\
\mathrm{C}_{3}, \mathrm{NH}_{2}\end{array}$ & 1.9 & 2.4 & \\
\hline 21.-23.01.1998 & 4.1 & 4.0 & 15.8 & Danish $1.5 \mathrm{~m}$ & DFOSC & $\begin{array}{l}\mathrm{CN}, \mathrm{NH}_{2}, \\
\mathrm{C}_{2}, \mathrm{C}_{3}\end{array}$ & 3.0 & 2.0 & \\
\hline 17.-19.03.1998 & 4.7 & 4.8 & 12.1 & Danish $1.5 \mathrm{~m}$ & DFOSC & $\begin{array}{l}\mathrm{CN}, \mathrm{C}_{2}, \\
\mathrm{C}_{3}, \mathrm{NH}_{2}\end{array}$ & 3.0 & 2.0 & \\
\hline 21./22.03.1998 & 4.7 & 4.8 & 12.0 & ESO $1.5 \mathrm{~m}$ & $\mathrm{~B} \& \mathrm{C}$ & $\begin{array}{l}\mathrm{CN}, \mathrm{C}_{2}, \\
\mathrm{C}_{3}, \mathrm{NH}_{2}\end{array}$ & 1.9 & 2.4 & \\
\hline 17./18.04.1998 & 5.0 & 5.2 & 11.2 & ESO $1.5 \mathrm{~m}$ & $\mathrm{~B} \& \mathrm{C}$ & $\begin{array}{l}\mathrm{CN}, \mathrm{C}_{2}, \\
\mathrm{C}_{3}\end{array}$ & 1.9 & 2.4 & non-photom. \\
\hline 7./8.05.1998 & 5.2 & 5.4 & 10.8 & Danish $1.5 \mathrm{~m}$ & DFOSC & - & & & clouds \\
\hline 20.-22.05.1998 & 5.3 & 5.5 & 10.5 & Danish $1.5 \mathrm{~m}$ & DFOSC & - & & & no Flats \\
\hline 20.-21.07.1998 & 5.9 & 6.0 & 9.6 & ESO $1.5 \mathrm{~m}$ & $\mathrm{~B} \& \mathrm{C}$ & - & & & clouds \\
\hline 5.-8.08.1998 & 6.0 & 6.2 & 9.4 & ESO $1.5 \mathrm{~m}$ & $\mathrm{~B} \& \mathrm{C}$ & $\mathrm{CN}$ & 1.9 & 1.6 & \\
\hline 23.-25.11.1998 & 7.0 & 7.0 & 8.0 & $3.6 \mathrm{~m}$ & EFOSC2 & $\mathrm{CN}, \mathrm{C}_{3}$ & 1.9 & 2.0 & \\
\hline 13.-15.01.1999 & 7.4 & 7.5 & 7.5 & $3.6 \mathrm{~m}$ & EFOSC2 & $\mathrm{CN}$ & 1.9 & 2.0 & \\
\hline 14./15.03.1999 & 7.9 & 8.0 & 7.2 & $3.6 \mathrm{~m}$ & EFOSC2 & $\mathrm{CN}$ & 1.9 & 2.0 & \\
\hline 12.-14.06.1999 & 8.6 & 8.6 & 6.7 & $3.6 \mathrm{~m}$ & EFOSC2 & - & & & clouds \\
\hline 12.-13.11.1999 & 9.8 & 9.9 & 5.7 & VLT/UT1 & FORS1 & $\mathrm{CN}$ & 5.3 & 2.0 & \\
\hline 5.-6.04.2000 & 10.8 & 10.8 & 5.3 & VLT/UT1 & FORS1 & & 5.3 & 2.0 & \\
\hline 28. -30.09 .2000 & 12.0 & 12.0 & 4.8 & VLT/UT1 & FORS1 & & 5.2 & 2.0 & \\
\hline 20.-26.01.2001 & 12.8 & 12.9 & 4.3 & VLT/UT1 & FORS1 & & 5.2 & 2.0 & \\
\hline
\end{tabular}

phenomenon) and can therefore not be used to derive cometary gas production rates. However, they are still useful to investigate the spatial coma distribution of the radicals observed. An extinction correction was performed using the standard extinction curve for La Silla and OHP, respectively. To estimate the error of the absolute flux calibration, spectra of standard stars were calibrated in the same way as the comet exposures, except for the sky background level, which could be derived from the long-slit star spectra directly. Calibrated standard stars from the photometric nights throughout the 5-year observing campaign have been compared to their catalog fluxes in the wavelengths interval $4500-5500 \AA$ (an interval covered by all 
Table 3. Fluorescence efficiencies ( $g$-factors) used for $\mathrm{C}_{2}$ and $\mathrm{C}_{3}$ radicals. The values correspond to $r_{\mathrm{h}}=1 \mathrm{AU}$ and scale proportionally to $r_{\mathrm{h}}^{-2}$.

\begin{tabular}{ccc}
\hline \hline Emission & $g$ & Reference \\
& {$\left[10^{-14} \mathrm{erg} \mathrm{s}^{-1}\right]$} & \\
\hline $\mathrm{C}_{2}(\Delta v=0)$ & 44.7 & Cochran et al. (1992) \\
$\mathrm{C}_{2}(\Delta v=1)$ & 24.0 & Cochran et al. (1992) \\
$\mathrm{C}_{3}$ & 38.0 & Cochran et al. (1992) \\
\hline
\end{tabular}

Table 4. Fluorescence efficiencies ( $g$-factors) used for the $\mathrm{NH}_{2}$ $(0,10,0)$ band. Computed $g$-factors from Kawakita et al. (2002) have been interpolated to the heliocentric distances of our observations. The values given are normalized to $r_{\mathrm{h}}=1 \mathrm{AU}$ and scale proportional to $r_{\mathrm{h}}^{-2}$. Negative values of $r_{\mathrm{h}}$ indicate pre-perihelion distances.

\begin{tabular}{rcc}
\hline \hline $\begin{array}{r}r_{\mathrm{h}} \\
{[\mathrm{AU}]}\end{array}$ & $\begin{array}{c}\mathrm{NH}_{2}: g / r_{\mathrm{h}}^{-2} \\
{\left[10^{-15} \mathrm{erg} \mathrm{s}^{-1}\right]}\end{array}$ & night \\
\hline-3.34 & 6.52 & $19 . / 20.08 .1996$ \\
-3.34 & 6.52 & $23 . / 24.08 .1996$ \\
-3.10 & 6.35 & $13 . / 14.09 .1996$ \\
-2.85 & 6.20 & $02 . / 03.10 .1996$ \\
-2.85 & 6.20 & $03 . / 04.10 .1996$ \\
2.89 & 6.21 & $30.9 . / 1.10 .1997$ \\
3.63 & 6.65 & $06 . / 07.12 .1997$ \\
3.63 & 6.75 & $07 . / 08.12 .1997$ \\
3.78 & 6.74 & $19 . / 20.12 .1997$ \\
4.13 & 6.77 & $20 . / 21.01 .1998$ \\
4.74 & 5.95 & $17 . / 18.03 .1998$ \\
4.74 & 5.95 & $21 . / 22.03 .1998$ \\
\hline
\end{tabular}

instrumental setup's and not influenced by the reflex using DFOSC at the $1.5 \mathrm{~m}$ Danish telescope). This comparison showed that deviations in absolute flux calibration throughout the observing campaign are less than $10 \%$. Of course, this error is only a lower limit of the errors in the comet spectra, because to measure cometary gas emissions, additional reduction steps are required.

To measure the intensity of the cometary gas emissions, the underlying continuum, caused by solar light scattered on dust particles, must be subtracted. The solar spectrum was taken from a catalog (Kurucz et al. 1984) and smoothed to the resolution of the comet observations by convolution with a Gaussian profile, followed by a polynomial fit to the cometary continuum flux level. The emission bands were then integrated to determine the band intensities, $F$. To convert to column densities, $N$, of the observed radical, the fluorescence efficiency factors ( $g$-factors) of the detected emission bands are used: $N=\frac{4 \pi F}{g \Omega}$. Here $\Omega$ denotes the solid angle determined by the pixel size and slit widths. The $g$-factors used are given in Tables 3 to 5 .

\section{Observed emission bands}

The exceptional activity of comet Hale-Bopp allowed us to observe emissions of gaseous coma species at heliocentric distances normally not accessible in comets. The sublimation of cometary ices depends on their volatility, the chemical composition of the nucleus and its physical structure. To constrain

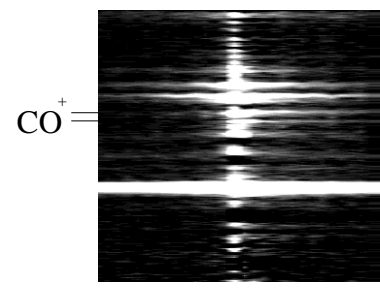

21.1.1998

$4.1 \mathrm{AU}$

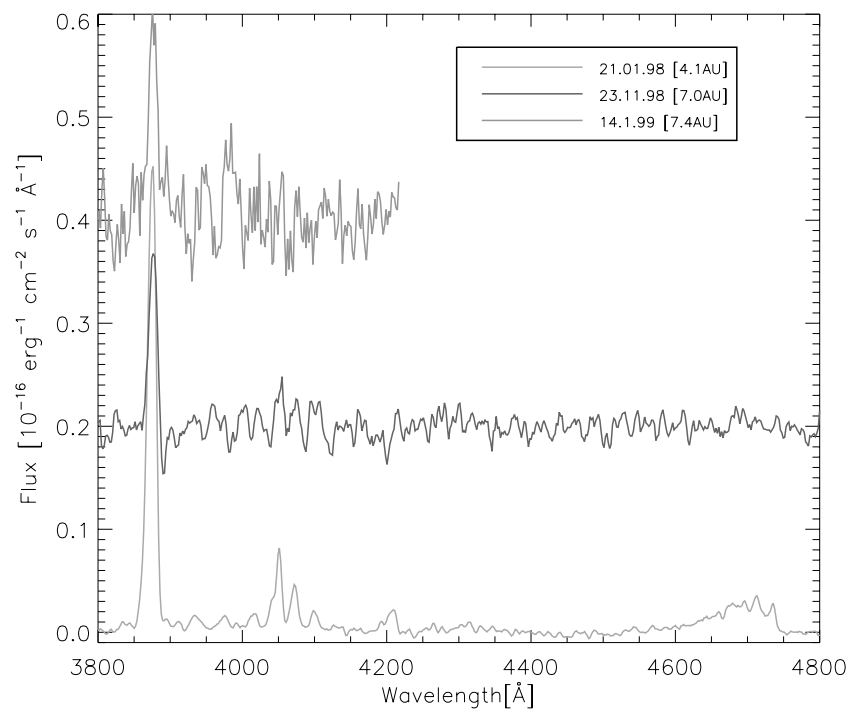

Fig. 2. Top left: spectrum obtained on January $21,1998\left(r_{\mathrm{h}}=4.1 \mathrm{AU}\right.$, $1200 \mathrm{~s}$ exposure time). The spectrum is flux calibrated and the underlying continuum caused by solar light scattered on dust particles has been subtracted. The horizontal direction covers approx. $6 \times 10^{5} \mathrm{~km}$ projected radial distance around the nucleus. The vertical dispersion direction covers 3800-4300 A. Top right: average of two spectra obtained on November, 25, $1998\left(r_{\mathrm{h}}=7.0 \mathrm{AU}, 2400 \mathrm{~s}\right.$ total exposure time). Spatial and wavelengths coverage as to the left. Both spectra were binned by a factor 10 in spatial direction. bottom: cuts through spectra obtained at different heliocentric distances, taken at about $7 \times 10^{4} \mathrm{~km}$ sunward. The spectrum at $4.1 \mathrm{AU}$ has been reduced by a factor of 5 in intensity. It shows emissions of $\mathrm{CN}$ at $3875 \AA$ and $4200 \AA, \mathrm{C}_{3}$ at $4000-4100 \AA$ and $\mathrm{C}_{2}$ at $4600-4750 \AA$. The spectra at 7.0 AU and 7.4 AU are offset in intensity for display. $\mathrm{C}_{3}$ is still detected at 7.0 AU, but not anymore at 7.4 AU.

this process, it is important to observe the activity of various species over a range of heliocentric distances. Although the parent molecules can not be observed in the optical range, the emissions of their daughter products seen in our spectra (Fig. 1) are indicators for activity of their parent molecules.

Emission bands of the $\mathrm{NH}_{2}$ radical $\left(\tilde{A}^{2} A_{1}-\tilde{X}^{2} B_{1}\right.$ system) were detected from 4.6 AU pre-perihelion up to 4.7 AU postperihelion (see Table 2). The emissions show a relatively weak intensity of bands starting at upper levels with odd vibrational quantum numbers (Rauer et al. 1997). It has recently been shown (Glinski et al. 2001; Kawakita et al. 2001a, 2001b, 2002) that this behaviour is consistent with fluorescence excitation and radiative relaxation in the ground state.

Emission bands of the $\mathrm{C}_{2}\left(d^{3} \Pi_{q}-a^{3} \Pi_{u}\right.$ Swan system at $5160 \AA)$ and $\mathrm{C}_{3}\left(\tilde{A}^{1} \Pi_{u}-\tilde{X}^{1} \Sigma_{g}^{+}\right.$system at $\left.4050 \AA\right)$ radicals were 

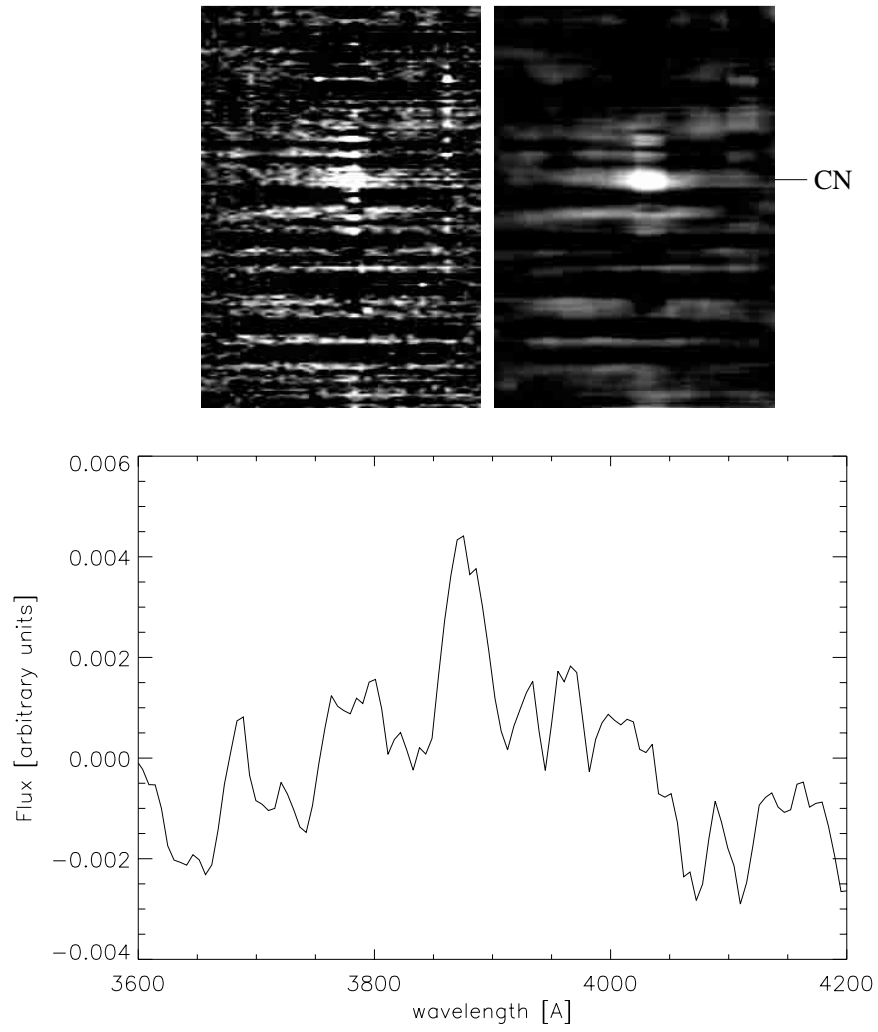

Fig. 3. Top left: average of 6 spectra of the $\mathrm{CN}(0,0)$ emission band obtained at $r_{\mathrm{h}}=9.8 \mathrm{AU}$ on November 13, 1999, using the VLT(UT1)/FORS1 (6100 s total exposure time). The underlying continuum caused by solar light scattered on dust particles has been subtracted. The horizontal direction covers a projected radial distance of $5 \times 10^{5} \mathrm{~km}$ around the nucleus. The vertical direction covers a wavelength range of $3600-4200 \AA$. The spectrum has been binned by a factor of 10 in spatial direction and a factor of 2 in the spectral direction. Top right: same spectrum as to the left, but binned further (factors 4 and 2 in spatial and spectral direction, respectively). The $\mathrm{CN}(0,0)$ emission band is clearly visible. Bottom: spectrum at $80000 \mathrm{~km} \mathrm{nu}-$ cleocentric distance, taken in top left spectrum and averaged by a factor 5 in wavelength. The $\mathrm{CN}$ emission band appears at $3875 \AA$.

recorded already at the start of this observing program, at 4.6 AU pre-perihelion (Rauer et al. 1997). The $\Delta v=0$ bands of $\mathrm{C}_{2}$ could be detected up to 5.0 AU post-perihelion (see Table 2). The $\mathrm{C}_{3}$ emission bands were, however, still detected at 7.0 AU (Fig. 2). The emission is weak, but allows to derive upper limits of the gas production rate at this large distance. Emission of $\mathrm{C}_{3}$ around $7 \mathrm{AU}$ has also been reported by Schleicher et al. (1999) in pre-perihelion observations.

Only few emissions of gaseous species have been observed in comets at distances beyond 5 AU. In comet 29/Schwassmann-Wachmann $1, \mathrm{CO}, \mathrm{CO}^{+}$and $\mathrm{CN}$ have been detected (e.g.: Cochran et al. 1991; Senay \& Jewitt 1994). In addition, a detection of $\mathrm{CN}$ emission in Chiron at 11.3 AU has been reported during an activity outburst (Bus et al. 1991). In comet Hale-Bopp, the $\mathrm{CN}\left(B^{2} \Sigma^{+}-X^{2} \Sigma^{+}\right)$violet system at $3880 \AA$ was first detected at $r_{\mathrm{h}}=6.8 \mathrm{AU}$ pre-perihelion (Fitzsimmons et al. 1996). In our observing campaign, we could detect the $\mathrm{CN}(0,0)$ emission band up to 9.8 AU postperihelion (Fig. 3).
Table 5. Fluorescence efficiencies ( $g$-factors) used for the CN $(0,0)$ emission band. The values are normalized to $r_{\mathrm{h}}=1 \mathrm{AU}$ and scale proportional to $r_{\mathrm{h}}^{-2}$. The $\mathrm{CN} g$-factors were computed in model calculations made by Schleicher (1983) to take into account the Swings effect. These calculations extend up to $r_{\mathrm{h}}=5 \mathrm{AU}$. Beyond this distance, only the scaling with solar flux has been taken into account (and thus the normalized value to $1 \mathrm{AU}$ is constant in this table). Negative values of $r_{\mathrm{h}}$ indicate pre-perihelion distances.

\begin{tabular}{rrcc}
\hline \hline $\begin{array}{r}r_{\mathrm{h}} \\
{[\mathrm{AU}]}\end{array}$ & $\begin{array}{r}\dot{r_{\mathrm{h}}}\left[\mathrm{km} \mathrm{s}^{-1}\right] \\
{\left[10^{-13} \mathrm{erg} \mathrm{s}^{-1}\right]}\end{array}$ \\
\hline-4.60 & -17.5 & 3.60 & night \\
-3.98 & -18.4 & 3.70 & $24 . / 27.04 .1996$ \\
-3.34 & -19.5 & 3.60 & $18 . / 19.08 .1996$ \\
-3.34 & -19.5 & 3.60 & $19 . / 20.08 .1996$ \\
-3.34 & -19.5 & 3.60 & $23 . / 24.08 .1996$ \\
-3.10 & -20.0 & 3.60 & $13 . / 14.09 .1996$ \\
-2.85 & -20.4 & 3.85 & $02 . / 03.10 .1996$ \\
-2.85 & -20.4 & 3.85 & $03 . / 04.10 .1996$ \\
3.51 & 19.2 & 3.08 & $23 . / 24.11 .1997$ \\
3.63 & 19.0 & 3.08 & $06 . / 07.12 .1997$ \\
3.63 & 19.0 & 3.08 & $07 . / 08.12 .1997$ \\
3.78 & 18.7 & 3.08 & $19 . / 20.12 .1997$ \\
4.13 & 18.1 & 3.21 & $20 . / 21.01 .1998$ \\
4.13 & 18.1 & 3.21 & $21 . / 22.01 .1998$ \\
4.13 & 18.1 & 3.21 & $22 . / 23.01 .1998$ \\
4.74 & 17.3 & 3.40 & $17 . / 18.03 .1998$ \\
4.74 & 17.3 & 3.40 & $21 . / 22.03 .1998$ \\
5.00 & 16.9 & 3.40 & $17 . / 18.04 .1998$ \\
6.00 & 15.6 & 3.73 & $05 . / 06.08 .1998$ \\
6.00 & 15.6 & 3.73 & $06 . / 07.08 .1998$ \\
7.00 & 14.7 & 3.73 & $23 . / 24.11 .1998$ \\
7.00 & 14.7 & 3.73 & $24 . / 25.11 .1998$ \\
7.40 & 14.3 & 3.73 & $13 . / 14.01 .1999$ \\
7.40 & 14.3 & 3.73 & $14 . / 15.01 .1999$ \\
7.90 & 13.9 & 3.73 & $14 . / 15.03 .1999$ \\
9.80 & 12.6 & 3.73 & $12 . / 13.11 .1999$ \\
10.81 & 12.1 & 3.73 & $05 . / 06.04 .2000$ \\
12.80 & 11.1 & 3.73 & $20 . / 21.01 .2001$ \\
12.80 & 11.1 & 3.73 & $24 . / 25.01 .2001$ \\
\hline & & & \\
\hline
\end{tabular}

Radio and infrared emissions of some of the suspected parent molecules of $\mathrm{CN}, \mathrm{C}_{2}, \mathrm{C}_{3}$ and $\mathrm{NH}_{2}$ have been detected in comet Hale-Bopp. But even in this bright comet, $\mathrm{HCN}(\mathrm{CN}$ parent) could only be detected up to $6.7 \mathrm{AU}$ at most (e.g. Biver et al. 1999). Emission of $\mathrm{C}_{2} \mathrm{H}_{2}$ and $\mathrm{C}_{2} \mathrm{H}_{6}$ (parent molecules of $\mathrm{C}_{2}$ ) have been observed (Dello Russo et al. 2001). $\mathrm{C}_{2} \mathrm{H}_{6}$ could be followed up to $3 \mathrm{AU}$. None of the proposed parent molecules of $\mathrm{C}_{3}$ have been detected in comets yet. Ammonia, the main parent molecule of $\mathrm{NH}_{2}$, can be detected in comets only around perihelion (e.g. Bird et al. 1997). The detected emissions of the daughter products, therefore, significantly extend the range over which activity can be accessed.

\section{Haser scale lengths}

Gas production rates are frequently derived using the Haser model (Haser 1957). The model assumes isotropic outgassing and expansion at constant speed and a 2-step exponential decay process leading from parent to daughter molecules and further 
to grand-daughter products. The number density of the daughter products can then be computed by:

$n(r)=\frac{Q}{4 \pi v r^{2}}\left(\frac{l_{\mathrm{d}}}{l_{\mathrm{p}}-l_{\mathrm{d}}}\right)\left(\mathrm{e}^{-\frac{r}{l_{\mathrm{p}}}}-\mathrm{e}^{-\frac{r}{l_{\mathrm{d}}}}\right)$.

Here, $v$ denotes the gas velocity, $l_{\mathrm{p}}$ and $l_{\mathrm{d}}$ are the parent and daughter scale lengths, respectively. $Q$ is the cometary gas production rate and $r$ the nucleocentric distance. Although the model is a crude simplification of the real coma processes, it allows the derivation of production rates in a simple way with few free parameters and because of its frequent use serves as a kind of reference model.

In order to compute gas production rates of comets from Eq. (1), the Haser scale lengths must be known. If we indeed assume isotropic expansion at constant velocity and decay by a dominant photodissociation process, the decay scale lengths of the parent and daughter molecules can be computed from the gas velocity and their respective lifetime, $\tau_{\mathrm{p}, \mathrm{d}}$, as $l_{\mathrm{p}, \mathrm{d}}=v \tau_{\mathrm{p}, \mathrm{d}}$. However, excess energies upon photodissociation, multiple parent molecules and the existence of various destruction pathways modify the scale lengths of an observed daughter species. The Haser scale lengths derived from measured spatial coma distributions therefore correspond to "effective" scale lengths (Combi \& Delsemme 1980). In general, these can not be converted to physical parameters without considering the dynamical and chemical coma processes in full detail.

Spatial radial column density profiles for the observed daughter products in terms of the Haser model can be computed by integrating Eq. (1) along the line of sight (Haser 1957). We have derived effective Haser scale lengths from the measured spatial column density profiles by finding the best fit of such computed profiles to the data. To obtain a maximum signal-tonoise-ratio $(S / N)$ on the column density distribution, all spatial profiles taken in the same night and the respective sunward and tailward directions were averaged before fitting the computed profiles. At the large heliocentric distances covered by our observations, the chemistry within the FOV is governed mainly by the decay of the parent molecules. This can be easily estimated by comparing the scale lengths extrapolated from values determined near $1 \mathrm{AU}$ (see Table 6) with the projected nucleocentric distance covered by the FOV of our observations. Dissociation of the daughter products dominates far outside the FOV. Keeping parent and daughter scale lengths as free parameters in the fitting process only barely constrains the derived daughter scale lengths, and, consequently, the daughter scale lengths can not be derived from our data. However, the resulting parent Haser scale lengths varied by less than $10 \%$, only for $\mathrm{NH}_{2}$ the differences increased to $15 \%$. To derive the parent Haser scale lengths given in Table 6, dissociation of the daughter products has been neglected, assuming that all dissociation occurs far outside the FOV. To further verify whether the effect of daughter molecule dissociation on the derived parent scale lengths is small, we set $l_{\mathrm{d}}$ to the smallest literature values given in Table 6. Again, the resulting parent Haser scale lengths agree with the values derived previously within $10 \%$ to $15 \%$. An example of the resulting spatial profiles and their fit to a set of measured fluxes is given in Fig. 4.
Up to now, Haser scale lengths have been mainly derived from observations of comets near $1 \mathrm{AU}$. They were unknown at large $r_{\mathrm{h}}$ and, therefore, were usually extrapolated by a power law. Photodissociation rates scale proportional to $r_{\mathrm{h}}^{2}$, and in most cases only this dependence of the photo chemical processes on solar flux is taken into account when analyzing cometary spectra. Haser scale lengths, therefore, are usually scaled simply as $r_{\mathrm{h}}^{2}$ (Table 6). However, the coma dynamics also varies with heliocentric distance. In comet HaleBopp coma gas velocities could be measured from molecular emissions at radio wavelengths up to $6.5 \mathrm{AU}$ and vary as $v=1.120( \pm 0.014) r_{\mathrm{h}}^{-0.41( \pm 0.01)} \mathrm{km} \mathrm{s}^{-1}$ (Biver et al. 1999). By simply combining the varying solar energy and gas velocity with distance, a variation of scale lengths proportional to about $r_{\mathrm{h}}^{1.6}$ would be expected. Here, we assume the formation of the observed daughter molecules is governed by a simple two-step photo-chemistry. In addition, it is assumed that the daughter products are thermalized to the velocity of the neutral gas and move with the same velocity as the parent molecules observed in the radio range. Deviations from this scaling law would indicate the presence of a more complex chemistry, or additional processes such as acceleration of the daughter product due to photo-excess energies (e.g. for $\mathrm{CN}$ ). We also note that comets with different production rates than Hale-Bopp may show different Haser scale lengths due to different gas expansion velocities and size of their collisional region.

The parent Haser scale lengths obtained from our data set are plotted versus heliocentric distance in Fig. 5. The scale lengths increase with increasing heliocentric distance. For $\mathrm{CN}$, Haser scale lengths could be derived up to 7.9 AU, but for the weak $\mathrm{NH}_{2}$ emission lines only data within 3.8 AU heliocentric distance could be used. The variation of Haser scale lengths with distance has been approximated by a power law: $a r_{\mathrm{h}} b_{\mathrm{p}}$ (Table 6). Haser scale lengths depend on the dynamical and chemical processes in the coma and can, therefore, strictly be used only for the heliocentric distance range at which they have been derived. For this reason, the Haser scale lengths and their scaling law given are valid only over the range of heliocentric distances used in our data set. We now discuss the scaling laws for the radicals observed.

\section{$\mathbf{N H}_{2}$}

The main parent molecule of $\mathrm{NH}_{2}$ is $\mathrm{NH}_{3}$, which is also the main N-bearing ice in comets (Bird et al. 1997, 1999). The main reaction path of the $\mathrm{NH}_{3}$ chemistry is photodissociation: $\mathrm{NH}_{3} \rightarrow \mathrm{NH}_{2} \rightarrow \mathrm{NH}$. Other reaction branches are of minor importance. We assume that the excess energy given to the daughter products is small. The derived scaling of the parent Haser scale lengths of $\mathrm{NH}_{2}$ of $r_{\mathrm{h}}^{1.5 \pm 0.9}$ is therefore in fairly good agreement with the value expected for photodissociation of $\mathrm{NH}_{3}$.

\section{$\mathbf{C}_{2}$ and $\mathbf{C}_{3}$}

The variation of Haser parent scale lengths with heliocentric distance of $\mathrm{C}_{3}$ and $\mathrm{C}_{2}$ differs significantly from the expectations assuming simple photo-chemistry of a single parent molecule and constant outflow velocity. The derived Haser parent scale lengths vary as $r_{\mathrm{h}}^{2.6 \pm 0.2}$ and $r_{\mathrm{h}}^{2.8 \pm 0.4}$, respectively. For $\mathrm{C}_{2}$, this is close to the scaling previously found by Cochran (1985) and Cochran et al. (1992) (Table 6). $\mathrm{C}_{2} \mathrm{H}_{2}$ and $\mathrm{C}_{2} \mathrm{H}_{6}$ 

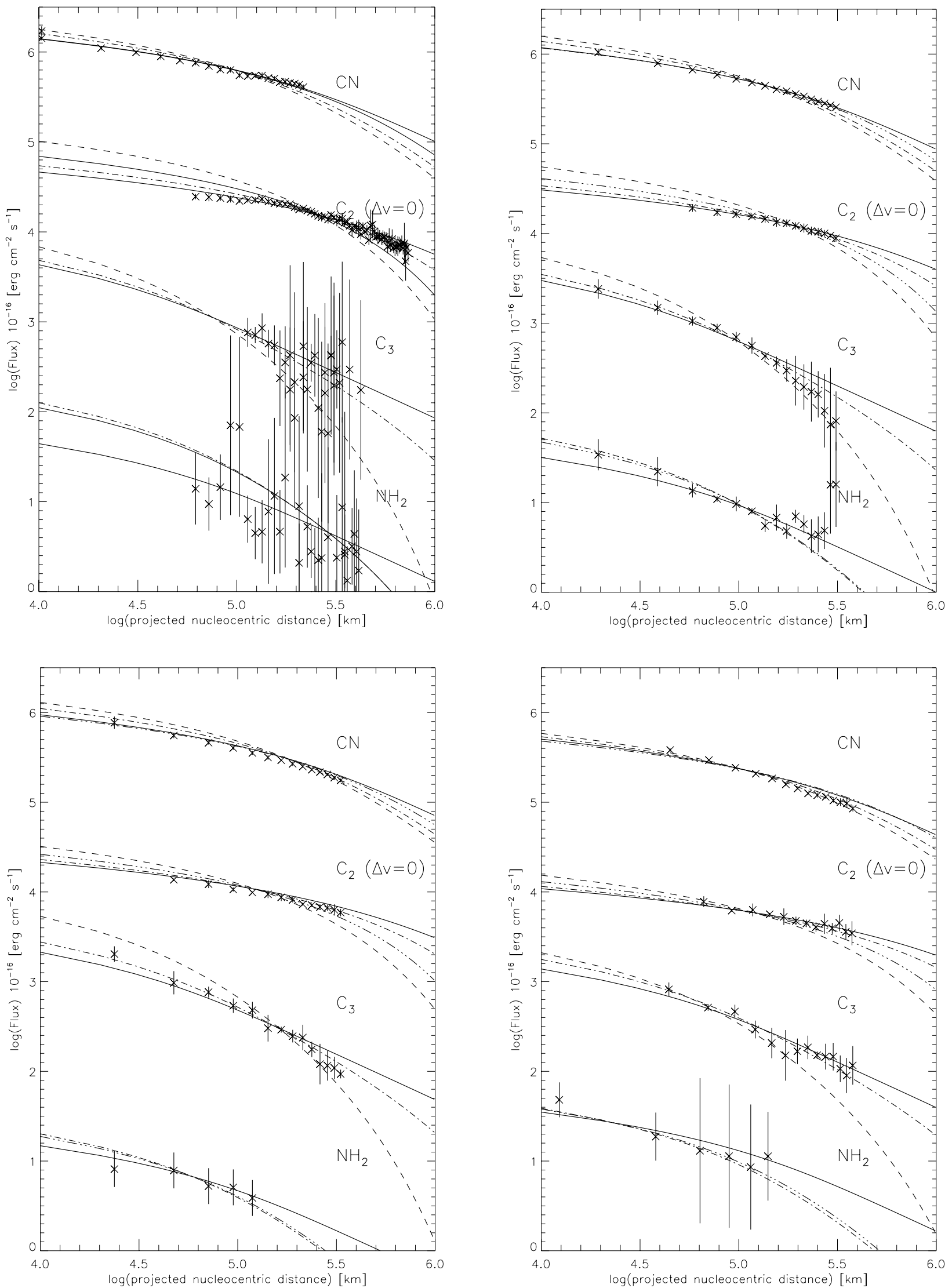

Fig. 4. Crosses: measured radial flux profiles; solid lines: fitted Haser profiles using scale lengths derived from this data set; dashed lines: Haser scale lengths from A'Hearn et al. (1995); dashed-dotted lines: Cochran et al. (1992); dashed-3dotted lines: Fink et al. (1991). Top left: Dec. 6, $1997\left(r_{\mathrm{h}}=3.6 \mathrm{AU}\right)$; top right: Dec. 19, $1997\left(r_{\mathrm{h}}=3.8 \mathrm{AU}\right)$; bottom left: Jan. 20, $1998\left(r_{\mathrm{h}}=4.1 \mathrm{AU}\right)$; bottom right: Mar. $21,1998\left(r_{\mathrm{h}}=4.7 \mathrm{AU}\right)$. The measured and the fitted Haser profiles of the various radicals have been offset vertically for display. 
Table 6. Parent and daughter Haser scale lengths, $l_{\mathrm{p}}$ and $l_{\mathrm{d}}$, at $1 \mathrm{AU}$ and their power law exponents $b_{\mathrm{p}}$ and $b_{\mathrm{d}}$. Haser scale lengths derived from values presented in this work are strictly valid only in the range $r_{\mathrm{h}} \geq 3 \mathrm{AU}$.

\begin{tabular}{llllll}
\hline \hline molecule & $l_{\mathrm{p}}[\mathrm{km}]$ & $b_{\mathrm{p}}$ & $l_{\mathrm{d}}[\mathrm{km}]$ & $b_{\mathrm{d}}$ & reference \\
\hline $\mathbf{C N}$ & $5.4 \pm 1.4 \times 10^{4}$ & $1.3 \pm 0.2$ & - & - & this work \\
& $1.3 \times 10^{4}$ & 2 & $2.2 \times 10^{5}$ & 2 & A'Hearn et al. (1995) \\
& $2.5 \times 10^{4}$ & 2 & $1.9 \times 10^{5}$ & 2 & Womack et al. (1994) \\
& $1.4 \times 10^{4}$ & 2 & $3.0 \times 10^{5}$ & 2 & Cochran et al. (1992) \\
& $1.5 \times 10^{4}$ & 2 & $1.9 \times 10^{5}$ & 2 & Randall et al. (1992) \\
& $2.8 \times 10^{4}$ & 2 & $3.2 \times 10^{5}$ & 2 & Fink et al. (1991) \\
& $1.8 \times 10^{4}$ & 2 & $4.2 \times 10^{5}$ & 2 & Newburn \& Spinrad (1989) \\
& $1.6 \times 10^{4}$ & 2 & $3.3 \times 10^{5}$ & 2 & Wyckoff et al. (1988) \\
\hline $\mathbf{C}_{3}$ & $1.3 \pm 0.4 \times 10^{3}$ & $2.6 \pm 0.2$ & - & - & this work \\
& $2.8 \times 10^{3}$ & 2 & $2.7 \times 10^{4}$ & 2 & A'Hearn et al. (1995) \\
& $3.1 \times 10^{3}$ & 2 & $15.0 \times 10^{4}$ & 2 & Cochran et al. (1985, 1992) \\
\hline $\mathbf{C}_{2}$ & $1.6 \pm 0.8 \times 10^{4}$ & $2.8 \pm 0.4$ & - & - & this work \\
& $2.2 \times 10^{4}$ & 2 & $6.6 \times 10^{4}$ & 2 & A'Hearn et al. (1995) \\
& $5.8 \pm 0.5 \times 10^{4}$ & 2 & $5.8 \pm 0.5 \times 10^{4}$ & 2 & Fink et al. (1991) \\
& $2.5 \times 10^{4}$ & 2.5 & $12.0 \times 10^{4}$ & 2 & Cochran et al. (1985, 1992) \\
\hline $\mathbf{N H}_{2}$ & $9.5 \pm 11.1 \times 10^{3}$ & $1.5 \pm 0.9$ & - & - & this work \\
& $4.9 \pm 1.5 \times 10^{3}$ & 2 & $6.9 \pm 2.0 \times 10^{3}$ & 2 & Fink et al. (1991) \\
& $4.1 \times 10^{3}$ & 2 & $62.0 \times 10^{3}$ & 2 & Cochran et al. (1985, 1992) \\
\hline
\end{tabular}

have been proposed as parent molecules of the $\mathrm{C}_{2}$ radical (Jackson 1976; Yamamoto 1981) and were recently detected in comets Hyakutake and Hale-Bopp (Mumma et al. 1996; Weaver et al. 1999). The chemical processes to form the $\mathrm{C}_{2}$ radical from these parents invoke several intermediate steps. In addition, other potential $\mathrm{C}_{2}$ parents (e.g. $\mathrm{CH}_{3} \mathrm{CN}, \mathrm{HC}_{3} \mathrm{~N}$ ) have been detected at radio wavelengths (Bockelée-Morvan et al. 2000). These need to be studied in a more complex model than a simple 2-step Haser model and are subject of future work.

The parent molecules of the $\mathrm{C}_{3}$ radical are still unknown. $\mathrm{C}_{3} \mathrm{H}_{4}$ (existing in two forms: $\mathrm{CH}_{3} \mathrm{CCH}$ (propyne) and $\mathrm{CH}_{2} \mathrm{CCH}_{2}$ (allene)) had been proposed on the basis that it is the most simple of carbon-chain molecules possibly forming $\mathrm{C}_{3}$ (Stief et al. 1972; Jackson 1976; Yamamoto 1981). The sometimes discussed isomer cyclopropene is an excited state of propene. Due to the inherent ring strain, this cyclic isomer undergoes readily ring-opening reactions, forming allene (Meijere et al. 2001). No $\mathrm{C}_{3}$ parent molecule has been detected in comets yet. The scaling of the derived Haser scale lengths indicates formation of $\mathrm{C}_{3}$ from a more complex chemistry, which again requires a more detailed chemical model.

Therefore, although the Haser model is able to approximate the measured column density profiles of $C_{2}$ and $C_{3}$ reasonably well, the production rates obtained do not reflect the sublimation rate of their parent molecules from the nucleus. For this purpose, a more complex chemical model has to be used, taking into account photo-processes, but also other possible reaction types. We have performed such modeling, which will be subject of a subsequent publication (Helbert et al., in preparation).

CN

The formation of the $\mathrm{CN}$ radical may be influenced by additional coma sources in comets near $1 \mathrm{AU}$. We therefore studied the formation of $\mathrm{CN}$ in more detail to investigate whether such additional sources may also play a role at large heliocentric distances.

The variation of $\mathrm{CN}$ Haser parent scale lengths with heliocentric distance is $r_{\mathrm{h}}^{1.3 \pm 0.2}$ (see Table 6). To investigate whether the derived parent Haser scale lengths of $\mathrm{CN}$ are in agreement with formation by photo-destruction of $\mathrm{HCN}$ molecules, we compare the observed parent scale lengths with theoretical values for HCN dissociation (Fig. 6). Equivalent parent Haser scale lengths were computed by applying a method suggested by Combi \& Delsemme (1980). The $\mathrm{CN}$ molecule receives an excess energy when formed by HCN photo-dissociation which has to be taken into account computing its expected parent scale length. An excess velocity of $1.06 \mathrm{~km} \mathrm{~s}^{-1}$ for $\mathrm{CN}$ has been used, as suggested by Bockelée-Morvan \& Crovisier (1985), who note, however, that this value is an upper limit since the poorly known conversion to rotational energy of the $\mathrm{CN}$ radical has been neglected. We assume a theoretical photo-dissociation rate of $\mathrm{HCN}$ of $1.5 \times 10^{-5} \mathrm{~s}^{-1}$ (Crovisier 1994). The gas velocity is assumed to vary as $1.120( \pm 0.014) r_{\mathrm{h}}^{-0.41( \pm 0.01)} \mathrm{km} \mathrm{s}^{-1}$, as measured in comet Hale-Bopp by Biver et al. (1999). The resulting effective $\mathrm{CN}$ Haser parent scale length shows fairly good agreement with the parent scale length of $\mathrm{CN}$ derived from our data in the range $r_{\mathrm{h}}>3 \mathrm{AU}$ (Fig. 6).

The resulting parent scale length extrapolated to $r_{\mathrm{h}}=1 \mathrm{AU}$ is larger than the parent scale lengths determined in comets observed near $1 \mathrm{AU}$, though a valid comparison should take account of the somewhat larger expansion velocities in comet Hale-Bopp (Combi et al. 1999). In any case, it is close to the scale length for photo-destruction of HCN computed from the HCN photo-dissociation lifetime, $\tau$, by assuming a constant gas velocity of $1 \mathrm{~km} \mathrm{~s}^{-1}$ and using the simple relation $l=\tau v$ (see Table 7). Considering the crude simplifications made by comparing these scale lengths with the measurements (see Table 6), 


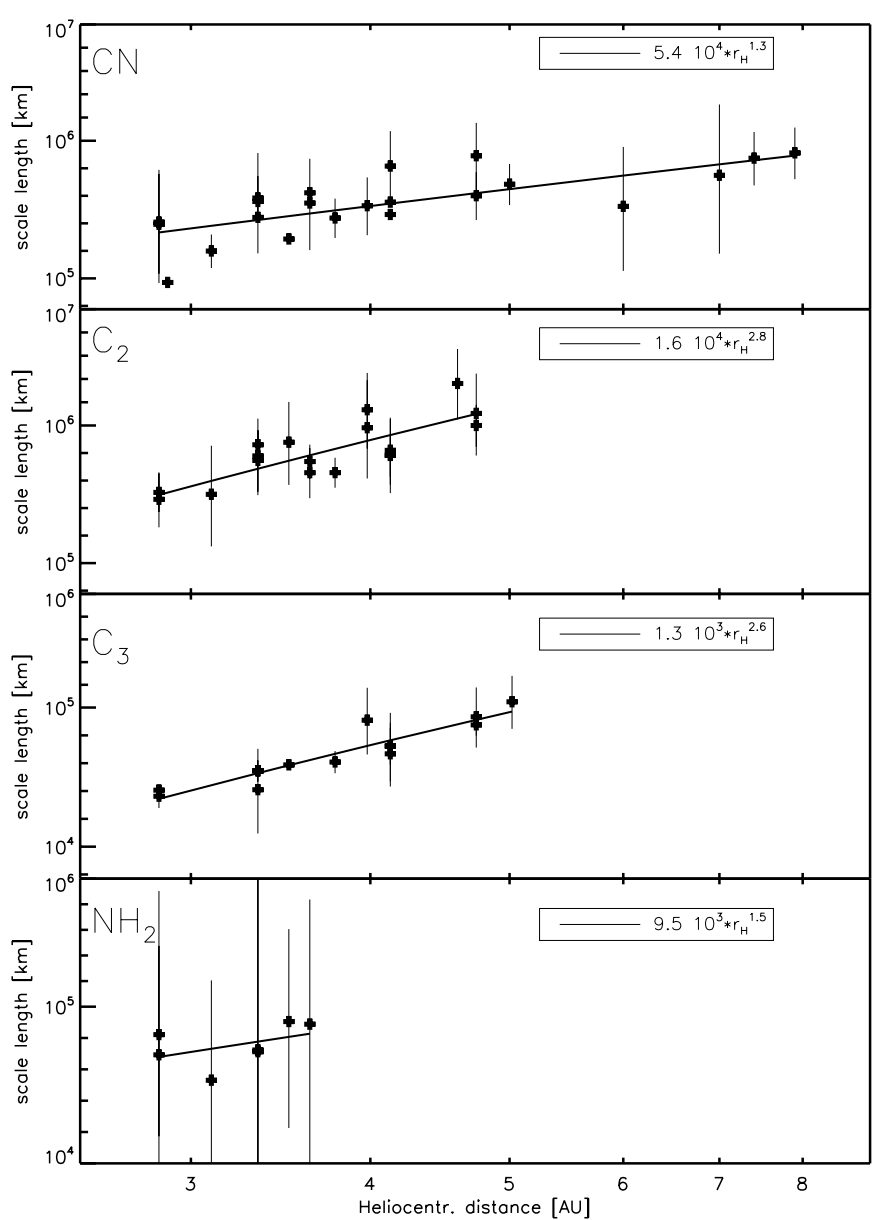

Fig. 5. Haser parent scale lengths versus heliocentric distance. Solid line: fit to data.

Table 7. Scale lengths, $l$, for photo-destruction of $\mathrm{CN}$ parent molecules derived from the respective dissociation lifetimes, $\tau$, assuming a constant gas velocity, $v$, of $1 \mathrm{~km} \mathrm{~s}^{-1}$ and using the simplified relation $l=\tau v$.

\begin{tabular}{lll}
\hline \hline parent & $\begin{array}{l}\text { scale length } \\
{[\mathrm{km}]}\end{array}$ & reference \\
\hline $\mathrm{CN}$ parent & $5.4 \pm 1.4 \times 10^{4}$ & this work \\
$\mathrm{HCN}$ & $6.7 \times 10^{4}$ & Crovisier (1994) \\
$\mathrm{HCN}$ & $7.7 \times 10^{4}$ & Huebner et al. (1992) \\
$\mathrm{HC}_{3} \mathrm{~N}$ & $1.5 \times 10^{4}$ & Crovisier (1994) \\
$\mathrm{HC}_{3} \mathrm{~N}$ & $2.6 \times 10^{4}$ & Huebner et al. (1992) \\
$\mathrm{CH}_{3} \mathrm{CN}$ & $1.5 \times 10^{5}$ & Crovisier (1994) \\
$\mathrm{HNCO}$ & $3.4 \times 10^{4}$ & Huebner et al. (1992) \\
\hline
\end{tabular}

the agreement is fairly good. Dissociation scale lengths of the other potential CN parent molecules listed in Table 7 are significantly lower or higher.

The variation of $\mathrm{CN}$ parent scale lengths and the extrapolated scale lengths at $1 \mathrm{AU}$ indicate that photo-destruction of $\mathrm{HCN}$ is the major formation process of $\mathrm{CN}$ in the data set used here. The differences of the extrapolated Haser parent scale lengths to $\mathrm{CN}$ parent scale lengths determined directly in comets near $1 \mathrm{AU}$ (Table 6) may indicate additional

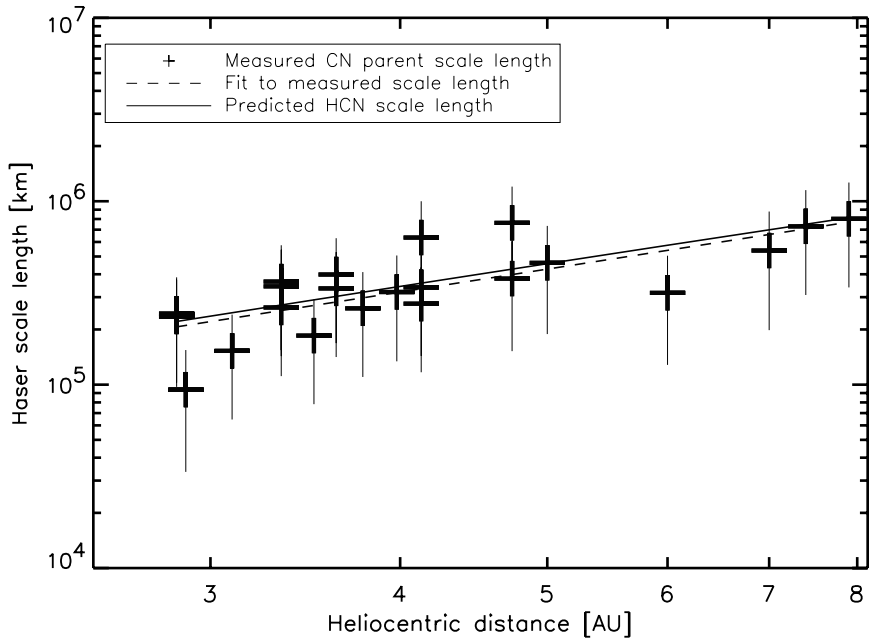

Fig. 6. Parent Haser scale lengths of $\mathrm{CN}$ versus heliocentric distance. Crosses: $\mathrm{CN}$ parent scale lengths derived from data; solid line: $\mathrm{CN}$ parent scale lengths computed from photodissociation of HCN (see text).

processes in comets near the Sun. However, further studies are required before definitive conclusions for comets near $1 \mathrm{AU}$ can be drawn.

\section{Production rates}

Production rates, $Q$, have been computed using the gas velocity by Biver et al. (1999) and Haser scale lengths derived in Sect. 4 . The production rates and the corresponding error from the fitting procedure are shown versus heliocentric distance in Fig. 7 and are given in Table 8.

Depending on the Haser scale lengths used, the derived production rates may differ. We investigated the quantitative differences in production rates computed using Haser scale lengths published in the literature and extrapolated to large $r_{\mathrm{h}}$, in comparison to the scale lengths derived from our data set (see Fig. 7). For $\mathrm{C}_{3}, \mathrm{C}_{2}$ and $\mathrm{NH}_{2}$, production rates can differ by up to a factor of 2, depending on which set of Haser scale lengths is used. For CN, production rates agree well up to about 4-5 AU, but the discrepancies increase somewhat at larger heliocentric distances. The scaling laws assumed for literature values and the scaling determined from our data differ. This causes increasing deviations in production rates with increasing heliocentric distance.

An additional influence on the derived production rates results from the gas velocity used and its assumed variation with heliocentric distance. When comparing with the literature, the same gas velocities must be used. Schleicher et al. compute their production rates with a constant gas velocity of $1 \mathrm{~km} \mathrm{~s}^{-1}$. Applying the velocity law from Biver et al. (1999), lowers the production rates in particular at large $r_{\mathrm{h}}$. A comparison to our measurements then shows good agreement between these observations (see Fig. 9 for $\mathrm{CN}$ ).

A'Hearn et al. (1995) introduced a classification scheme based on optical photometric observations of a large number of comets. The main difference in cometary production rate ratios appeared in the $\mathrm{C}_{2} / \mathrm{CN}$ ratio, which indicated a class of 
Table 8. Production rates computed using a Haser model. The Haser scale lengths used have been derived in this work (see Table 6). Negative values for $r_{\mathrm{h}}$ correspond to pre-perihelion distance.

\begin{tabular}{|c|c|c|c|c|c|}
\hline date & $\begin{array}{r}r_{\mathrm{h}} \\
{[\mathrm{AU}]}\end{array}$ & $\begin{array}{r}\mathrm{CN} \\
{\left[10^{25} \mathrm{~s}^{-1}\right]}\end{array}$ & $\begin{array}{r}\mathrm{C}_{2} \\
{\left[10^{25} \mathrm{~s}^{-1}\right]}\end{array}$ & $\begin{array}{r}\mathrm{C}_{3} \\
{\left[10^{25} \mathrm{~s}^{-1}\right]}\end{array}$ & $\begin{array}{r}\mathrm{NH}_{2} \\
{\left[10^{25} \mathrm{~s}^{-1}\right]}\end{array}$ \\
\hline $25 / 26.04 .1996$ & -4.60 & & $44.4 \pm 13.6$ & & $22.2 \pm 0.1$ \\
\hline 26/27.04.1996 & -4.60 & $20.0 \pm 0.1$ & $23.6 \pm 0.1$ & & \\
\hline $24 / 25.06 .1996$ & -3.98 & $35.8 \pm 5.4$ & $46.3 \pm 3.0$ & $1.7 \pm 0.6$ & $8.5 \pm 0.1$ \\
\hline 25/26.06.1996 & -3.98 & $64.6 \pm 13.8$ & $58.4 \pm 12.0$ & & $36.7 \pm 5.6$ \\
\hline $19 / 20.08 .1996$ & -3.34 & $78.7 \pm 33.1$ & $47.9 \pm 19.1$ & $9.1 \pm 2.1$ & $6.2 \pm 0.2$ \\
\hline 18/19.08.1996 & -3.34 & $79.9 \pm 0.1$ & $56.5 \pm 14.3$ & $9.2 \pm 0.1$ & \\
\hline 23/24.08.1996 & -3.34 & $124.9 \pm 24.1$ & $98.9 \pm 20.8$ & $11.6 \pm 0.1$ & $60.3 \pm 19.9$ \\
\hline $13 / 14.09 .1996$ & -3.10 & $197.1 \pm 25.3$ & $141.4 \pm 21.6$ & & $102.5 \pm 12.9$ \\
\hline 02/03.10.1996 & -2.85 & $183.8 \pm 64.6$ & $203.6 \pm 29.1$ & $27.2 \pm 10.2$ & $59.6 \pm 1.1$ \\
\hline 03/04.10.1996 & -2.85 & $190.4 \pm 42.8$ & $183.3 \pm 40.7$ & $33.8 \pm 10.0$ & $86.4 \pm 36.2$ \\
\hline 30/30.10.1997 & 2.89 & $102.3 \pm 0.3$ & $94.6 \pm 0.1$ & & $54.4 \pm 22.1$ \\
\hline 07/08.12.1997 & 3.63 & $29.5 \pm 0.3$ & $28.9 \pm 7.8$ & & $14.4 \pm 3.5$ \\
\hline 06/07.12.1997 & 3.63 & $37.2 \pm 7.2$ & $34.9 \pm 12.0$ & $6.9 \pm 0.1$ & $19.7 \pm 2.6$ \\
\hline 19/20.12.1997 & 3.78 & $26.0 \pm 4.7$ & $24.1 \pm 5.9$ & $4.7 \pm 0.9$ & $15.4 \pm 2.2$ \\
\hline 21/22.01.1998 & 4.13 & $25.4 \pm 4.4$ & $24.6 \pm 9.1$ & $5.1 \pm 3.1$ & \\
\hline 20/21.01.1998 & 4.13 & $29.8 \pm 6.8$ & $27.9 \pm 5.9$ & $6.7 \pm 3.1$ & $9.5 \pm 0.9$ \\
\hline 22/23.01.1998 & 4.13 & $30.0 \pm 2.3$ & $31.2 \pm 6.5$ & $11.5 \pm 0.1$ & \\
\hline $17 / 18.03 .1998$ & 4.74 & $18.9 \pm 2.3$ & $14.5 \pm 6.8$ & $7.1 \pm 6.4$ & $2.2 \pm 0.6$ \\
\hline $21 / 22.03 .1998$ & 4.74 & $19.0 \pm 0.1$ & $21.1 \pm 5.5$ & $4.8 \pm 0.1$ & $28.5 \pm 19.4$ \\
\hline 06/07.08.1998 & 6.00 & $4.9 \pm 1.3$ & & & \\
\hline 23/24.11.1998 & 7.00 & $3.3 \pm 0.1$ & & & \\
\hline $24 / 25.11 .1998$ & 7.00 & $4.4 \pm 1.1$ & & $\leq 0.63$ & \\
\hline 14/15.01.1999 & 7.40 & $3.1 \pm 0.2$ & & & \\
\hline 13/14.01.1999 & 7.40 & $3.7 \pm 1.1$ & & & \\
\hline $14 / 15.03 .1999$ & 7.90 & $2.9 \pm 0.1$ & & & \\
\hline 12/13.11.1999 & 9.80 & $0.86 \pm 0.15$ & & & \\
\hline 05/06.04.2000 & 10.81 & $<1.8$ & & & \\
\hline $29 / 30.09 .2000$ & 12.00 & $<0.16$ & & & \\
\hline 20/21.01.2001 & 12.80 & $<0.06$ & & & \\
\hline $24 / 25.01 .2001$ & 12.80 & $<0.05$ & & & \\
\hline
\end{tabular}

carbon-depleted comets. These comets appeared also depleted in $\mathrm{C}_{3}$, but not in $\mathrm{NH}$. The production rate ratios of comet HaleBopp obtained from our data set are shown in Fig. 8. The $\mathrm{C}_{2} / \mathrm{CN}$ ratio confirms the classification of comet Hale-Bopp as "typical" comet. No significant variation with heliocentric distance has been found. The classification of comet Hale-Bopp is further supported by the $\mathrm{C}_{3} / \mathrm{CN}$ ratio. It does not change significantly when the Haser scale lengths published by A'Hearn et al. (1995) are taken (which strictly needs to be done to compare with similar models). A'Hearn et al. (1995) found no indication for differences in the $\mathrm{NH} / \mathrm{CN}$ ratio among the comets studied. The NH emission bands were not covered in our data set. Assuming that dissociation of $\mathrm{NH}_{2}$ is the main formation reaction of $\mathrm{NH}$, we can use the $\mathrm{NH}_{2} / \mathrm{CN}$ ratio to compare with the results by A'Hearn et al. to first order. Figure 8 indicates that the $\mathrm{NH}_{2} / \mathrm{CN}$ ratio in comet Hale-Bopp is again in agreement with most comets.

\section{Comparing $\mathrm{CN}$ and $\mathrm{HCN}$ production rates}

The production rates of coma molecules can be used to probe and improve cometary sublimation models and therefore aid in better understanding the nucleus composition and structure. However, if the observed molecule is a daughter product, such as $\mathrm{CN}$, its chemistry and formation mechanisms must be well understood to allow for the derivation of parent production rates. In particular, the parent molecules must be known. While it is widely accepted that most $\mathrm{CN}$ is produced from $\mathrm{HCN}$, extended coma sources (additional parent molecules, sublimation from organic grains, etc.) releasing $\mathrm{CN}$, or even $\mathrm{HCN}$, have been proposed as an additional major source in comets near $1 \mathrm{AU}$ in the past (e.g. Bockelée-Morvan \& Crovisier 1985; Crovisier \& Schloerb 1991). The source of $\mathrm{CN}$ is, therefore, still somewhat controversial. Several parent molecules, in addition to $\mathrm{HCN}$, have been detected in comets Hyakutake and Hale-Bopp. However, their abundances relative to water are low: $\mathrm{HC}_{3} \mathrm{~N}$ : $0.021 \%, \mathrm{CH}_{3} \mathrm{CN}$ : $0.02 \%$ (Bockelée-Morvan et al. 2000). For comparison, $\mathrm{HCN}$ abundances are on the order of $0.2 \%$ (Biver et al. 1999). Another recently discovered molecule in cometary comae is HNC. It is at present unclear whether $\mathrm{HNC}$ is a parent ice in the nucleus, created in the coma by chemical reactions, or a mixture of both.

In general, observations of $\mathrm{CN}, \mathrm{HCN}$ and other $\mathrm{CN}$-bearing molecules are made at heliocentric distances near $1 \mathrm{AU}$. The question of additional coma sources at larger heliocentric distances has been addressed in a first analysis by Rauer et al. (1997), by comparing CN production rates in comet Hale-Bopp in the range of 2.9-4.6 $\mathrm{AU}$ pre-perihelion to the production 


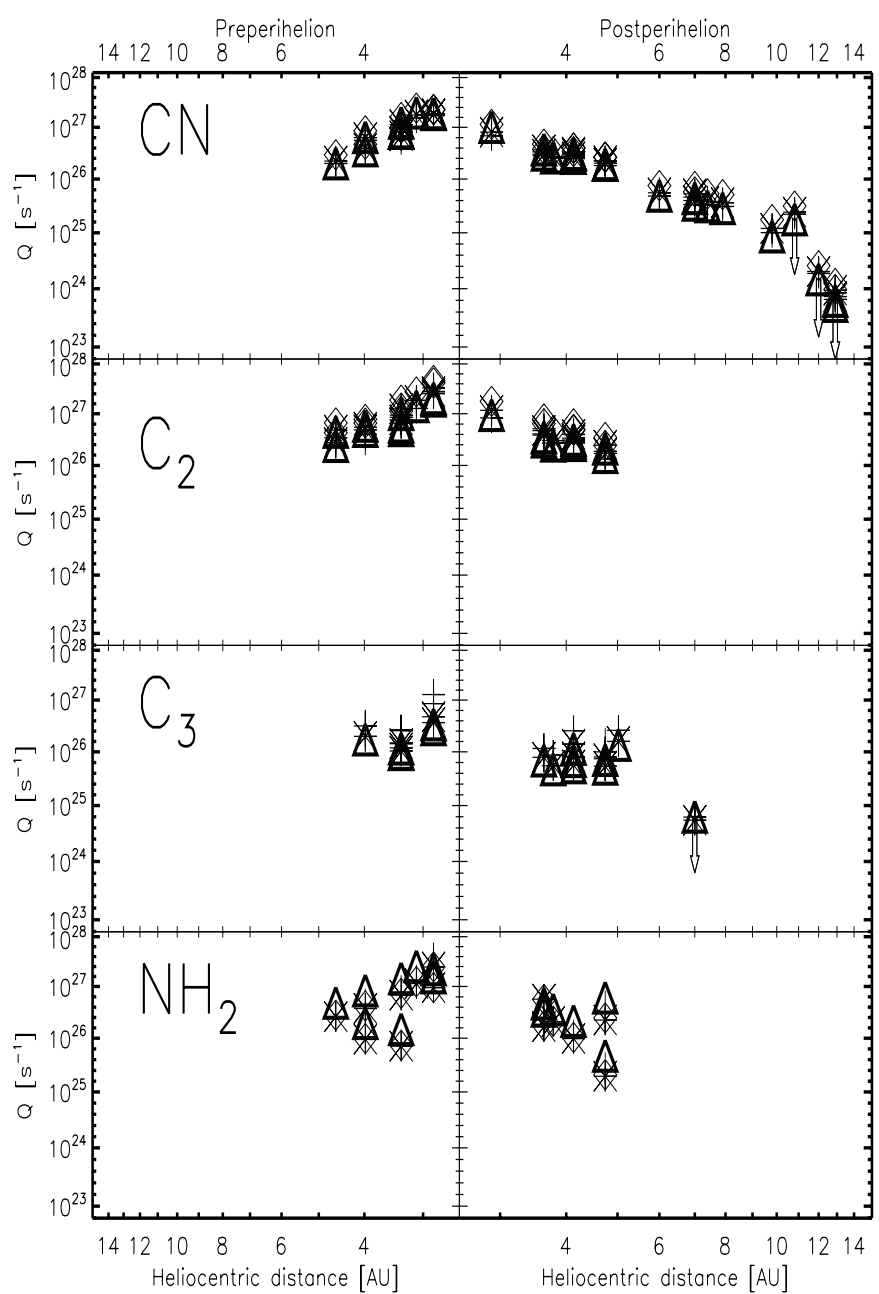

Fig. 7. Production rates versus heliocentric distance computed using different Haser scale lengths taken from: crosses: A'Hearn et al. (1995); stars: Cochran et al. (1992); diamonds: Fink et al. (1995); triangles: this work.

rate of HCN from radio observations (Biver et al. 1997, 1999). Agreement within the measurement uncertainties was found. Here, we pursue this study by determining gas production rates using the Haser parent scale lengths determined directly from our data set at large $r_{\mathrm{h}}$ and including post-perihelion observations.

In Fig. 9 we compare the $\mathrm{CN}$ production rates determined from our data set to the $\mathrm{HCN}$ production rates derived from radio observations (Biver et al. 1999). Both rates agree very well over a range of heliocentric distances. Together with the agreement of CN Haser parent scale length with photodissociation processes of $\mathrm{HCN}$, the measured $\mathrm{CN}$ production rates strongly indicate that formation of $\mathrm{CN}$ is dominated by $\mathrm{HCN}$ molecule dissociation at the heliocentric distances covered here.

\section{Comparison to a simple sublimation model}

The variation of gas production rates, $Q$, of the icy cometary constituents with changing heliocentric distance, $r_{\mathrm{h}}$, depends critically on the details of the sublimation processes of the cometary nucleus. The parameters determining cometary ice

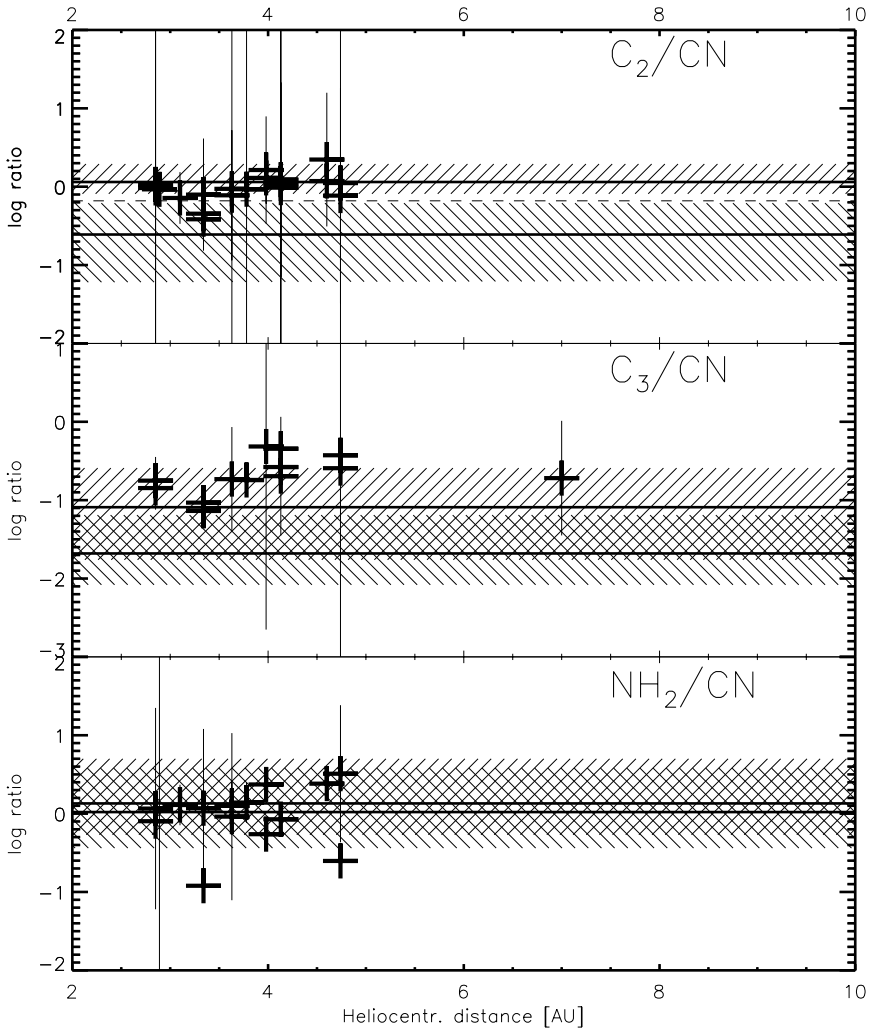

Fig. 8. $\log$ (ratio) of production rates versus heliocentric distance computed using Haser scale lengths derived from this data set. Solid lines denote the mean value as given by A'Hearn et al. (1995) for "typical" and C-depleted comets. The range given for both classes is indicated as right hatches ("typical") and left hatches (C-depleted).

sublimation are only poorly known. The chemical abundances, as well as the physical parameters of the nucleus, such as heat conductivity, porosity, amorphous or crystalline ice, the existence of a possible dust crust, etc., all have an influence on the level of gas sublimation of the various ices, and on their activity variation with heliocentric distance. Ices sublimating from the nucleus surface will result in gas activity variations different from those resulting from sublimation through pores from the nucleus interior (Benkhoff \& Huebner 1995, 1996; Benkhoff \& Boice 1996; Enzian et al. 1997). One way to verify the predictions of sublimation models and to constrain their poorly known input parameters is to observe gas production rates of ices of different volatility over a wide range of heliocentric distances.

In case of pure ice surfaces without internal heat conductivity, sublimation starts when sufficient solar energy is available. The corresponding heliocentric distance can be defined as the "turn-on" (or "turn-off") distance for sublimation. An important motivation to follow comet Hale-Bopp over a large heliocentric distance range was to observe the "turn-off" of activity. For $\mathrm{H}_{2} \mathrm{O}$, it occured at about 3-4 AU. For the more volatile species, "turn-off" appears at much larger distances. In our observations, the daughter radicals seen in the optical are used as indicators for the sublimation of their parents.

Although observations of $\mathrm{NH}_{2}$ can extend the accessible range of its parent $\mathrm{NH}_{3}$ much beyond the directly detectable 
range in comets, its emission bands are still too weak to be followed up to heliocentric distances where the turn-off of $\mathrm{NH}_{3}$ would be expected. Similarly, the likely parent molecules of $\mathrm{C}_{2}, \mathrm{C}_{2} \mathrm{H}_{2}$ and $\mathrm{C}_{2} \mathrm{H}_{6}$ are more volatile than water, and their turnoff in case of pure ice surfaces would be expected far outside the detectable range, even in a bright comet such as Hale-Bopp. The detection of the $\mathrm{C}_{3}$ radical around $7 \mathrm{AU}$ heliocentric distance indicates that its parent molecule(s) must be also among the most volatile species in comets.

For $\mathrm{CN}$, a daughter product of $\mathrm{HCN}$, the drop of activity as a result of decreasing solar energy flux would have been expected at about $10 \mathrm{AU}$ for pure ice without heat conductivity. This is within the detectable range of $\mathrm{CN}$ emission in comet Hale-Bopp. If sublimation of HCN occurs from the interior and thermal lag of the nucleus plays a significant role, activity may sustain up to larger heliocentric distances, at least post-perihelion. Observations of $\mathrm{CN}$ emission bands at large heliocentric distances, therefore, can provide observational evidence to constrain whether sublimation arises near the nucleus surface, or from the interior. We compare our observations to models of sublimation from a pure ice surface.

\subsection{The thermal model}

Our simplified one-dimensional model assumes a porous body, containing only one ice component (HCN). The body's porous structure is modeled as a bundle of tubes with a fixed tortuosity, but with pore diameters which change because of recondensation of the inward flowing gas. Heat is transferred into the interior of the body by solid-state heat conduction of the ice matrix, by vapor flow through the porous matrix (the flow being driven by a vapor pressure gradient), and by the transportation of latent heat released when hot vapor recondenses at colder areas inside the nucleus. The model can solve the mass and energy equations for different volatiles simultaneously in a $20 \mathrm{~m}$ thick surface shell of the body, although this feature is not used for this first analysis where only a pure ice surface is studied. The surface temperature is calculated from the balance between the net incoming solar flux, losses from thermal reradiation, heat needed for sublimation or becoming free during condensation, and heat transport in and out of the nucleus. For the boundary condition at the bottom of the shell we assume a constant heat flux and zero mass flux. A detailed descripton of our model can be found in Benkhoff \& Huebner $(1995,1996)$.

In the present model we assume a spherical model comet nucleus in the orbit of Comet Hale-Bopp with semi-major axis $a=185.55 \mathrm{AU}$, eccentricity $\epsilon=0.99507, \omega=133$, a spin axis perpendicular to the orbit plane, and a spin period of 11.5 hours. (Further parameters: ice composition always $100 \% \mathrm{HCN}$; pore radius $0.001 \mu \mathrm{m}$; initial temperature $10 \mathrm{~K}$; porosity 0.5 ; IR emissivity 0.96 ; and mean albedo 0.04 ). The results are calculated for a point located at the equator. The model calculations were carried out as follows: We started with a constant temperature $(T=10 \mathrm{~K})$ and a constant mass density distribution. Due to the energy input from the Sun the model comet increases its internal energy and sublimates its volatile components. From our calculations we obtain the temperature distribution in the interior, porosity and pore size distribution as a function of depth, the gas flux into the interior, and the gas flux of the nucleus into the coma at various positions of the comet in its orbit around the Sun. To compare with the measured $\mathrm{CN}$ and $\mathrm{HCN}$ production rates and constrain the influence of thermal lag on the comet's activity, we perform our simulations for a range of thermal conductivities $\left(0.000-0.568 \times 1 / T \mathrm{Wm}^{-1} \mathrm{~K}^{-1}\right)$.

\subsection{Comparison to measured gas production}

We simulate HCN sublimation from pure ice on the nucleus surface using the thermal model described above. For example, we could imagine the extreme scenario that $\mathrm{HCN}$ ice could exist in patches on the nucleus surface. Although this assumption is not considered as a realistic view of the nucleus composition and structure, in a first approach to use measured gas production rates to constrain nucleus sublimation models we have to investigate whether such extreme scenarios are supported, or ruled out, by the observations.

The internal heat conductivity of the nucleus is one of the poorly known parameters influencing the sublimation process. Figure 9 shows the measured production rates of $\mathrm{CN}$ and of HCN from radio observations. The data points are compared to computed $\mathrm{HCN}$ production rates for various heat conductivities $\left(k=0,0.005,0.05 \mathrm{Wm}^{-1} \mathrm{~K}^{-1}\right)$. This comparison implies an extremely low heat conductivity (much less than $0.005 \mathrm{Wm}^{-1} \mathrm{~K}^{-1}$ ) of cometary material. The measurements even agree with the extreme assumption of HCN sublimation from the nucleus surface and no heat conduction into the interior.

Therefore, a nucleus structure in agreement with the observations would consist of material of very low heat conduction and sublimation of $\mathrm{HCN}$ ice at or near the cometary surface. However, we can not completely rule out $\mathrm{HCN}$ sublimation from the interior. If heat conduction to the interior would be very high, the sublimation front of $\mathrm{HCN}$ ice would also respond quickly to the changing solar energy input, and the resulting variation of gas production rate with heliocentric distance would be similar to pure ice surfaces. Additional information is required to distinguish between these two cases. For example, the quantitative comparison to production rate ratios for different ices may provide such additional constraints. However, the required more detailed model analysis is beyond the scope of this paper.

From the simple analysis performed here, we can constrain the nucleus parameters to such cases where the sublimation of ices is governed by fast reaction to the variation of solar energy input along the comet's orbit, and no strong thermal lag of the nucleus.

\section{Summary}

Comet Hale-Bopp provided the opportunity to observe the gas activity of a comet at heliocentric distances from 4.6 AU preperihelion to $12.8 \mathrm{AU}$ post-perihelion. Emission bands of $\mathrm{CN}$, $\mathrm{C}_{3}, \mathrm{C}_{2}$ and $\mathrm{NH}_{2}$ have been observed in the monitoring campaign presented here. The $\mathrm{CN}$ emission band at $3875 \AA$ could 


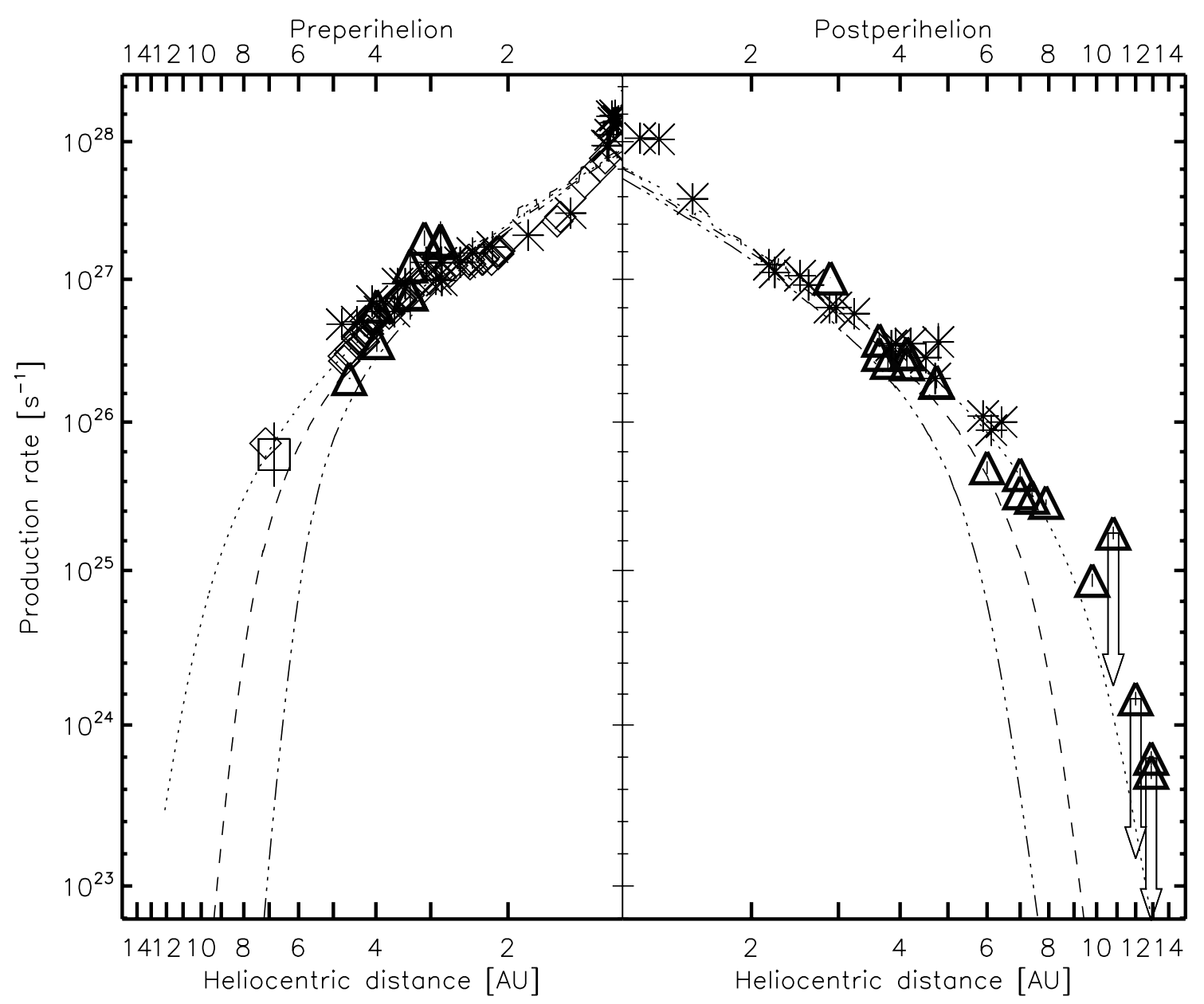

Fig. 9. Production rates of CN (triangles: this work, triangles with arrows indicate upper limits; diamonds: Schleicher et al. 1997, modified with velocity dependence of Biver et al. 1999; square: Fitzsimmons et al. 1996). Stars indicate HCN production rates taken from Biver et al. (1999). Sublimation of HCN ice versus heliocentric distance has been computed for various heat conductivities, $k$, for comparison (dotted line: $k=0 \mathrm{Wm}^{-1} \mathrm{~K}^{-1}$; dashed line: $k=0.005 \mathrm{Wm}^{-1} \mathrm{~K}^{-1}$; dashed-dotted line: $\left.k=0.05 \mathrm{Wm}^{-1} \mathrm{~K}^{-1}\right)$. The model results have been scaled by a constant scaling factor to fit the data points.

be detected up to $9.8 \mathrm{AU}$ heliocentric distance post-perihelion. $\mathrm{C}_{3}$ emission was still detected at 7.0 $\mathrm{AU}$, while $\mathrm{C}_{2}$ and $\mathrm{NH}_{2}$ could not be followed beyond 5.0 AU and 4.7 AU, respectively.

The following results have been obtained:

1. Effective Haser parent scale lengths for the observed radicals have been derived, providing scale lengths for $r_{\mathrm{h}}>3$ AU (Table 6).

2. At the heliocentric distances studied here, the formation of the $\mathrm{CN}$ radical is dominated by photodissociation of $\mathrm{HCN}$. No indications for other potential sources of $\mathrm{CN}$ have been found. This conclusion is based on the following results:

- Haser parent scale lengths of the $\mathrm{CN}$ radical derived from our data are in very good agreement with the HCN scale lengths for photo-dissociation.

- The measured $\mathrm{CN}$ production rates are in good agreement with HCN production rates (Biver et al. 1999) measured at radio wavelengths.

The agreement found with formation from HCN leaves little room for additional major sources of $\mathrm{CN}$. Therefore, observations of $\mathrm{CN}$ emission bands can indeed be used to determine the $\mathrm{HCN}$ gas activity in distant comets. Due to its high fluorescence efficiency, the $\mathrm{CN}$ emission allows to extend the range of distances where $\mathrm{HCN}$ sublimation in comets can be studied significantly.

3. The gas production rates of $\mathrm{CN}$ and $\mathrm{HCN}$ have been compared to simple HCN sublimation models. The data are consistent with sublimation of HCN responding quickly to the change of solar energy input. Our observations, therefore, constrain the sublimation process to a position of the sublimation front within reach of the orbital heat wave and little thermal lag of the nucleus. A possible scenario is $\mathrm{HCN}$ sublimation at or near the nucleus surface with extremely low heat conduction into the nucleus interior.

In view of the large number of unkowns needed to model the sublimation process of cometary nuclei, constraints from gas activity observations are clearly needed. However, providing stringent constraints is difficult and only a successive process, starting by testifying the most simple and extreme assumptions, may finally lead to progress in our understanding of the nucleus interior. In this work, we have started by comparing sublimation of a pure $\mathrm{HCN}$ ice surface to the $\mathrm{HCN}$ and $\mathrm{CN}$ gas activity for a range of heat conductions into the nucleus interior. 
The data show that monitoring of the gas production rates of minor species can indeed provide constraints to cometary gas sublimation models. However, it is important to cover a wide range of heliocentric distances, in particular large distances where the sublimation is expected to be significantly influenced by the internal physical parameters of the nucleus, e.g. heat conduction processes. In view of the difficulties to detect gas emission at such large distances (beyond $8 \mathrm{AU}$ ), optical observations of $\mathrm{CN}$ are specially suited to attack this problem because of their high fluorescence efficiency and the volatility of its parent, $\mathrm{HCN}$.

Acknowledgements. Part of this work was supported by an ESA external fellowship (H. Rauer) and the German Deutsche Forschungsgemeinschaft, $D F G$ project number Ra 714/2-1,3.

\section{References}

A'Hearn, M. F., Millis, R., Schleicher, D. G., Osip, D. J., \& Birch, P. V. 1995, Icarus, 118, 223

Benkhoff, J., \& Huebner, W. F. 1995, Icarus, 114, 348

Benkhoff, J., \& Boice, D. C. 1996, Planet. Space Sci., 44, 665

Benkhoff, J., \& Huebner, W. F. 1996, Planet. Space. Sci., 44, 1005

Bird, M. K., Huchtmeier, W. K., Gensheimer, P., et al. 1997, A\&A, 325, L5

Bird, M. K., Janardhan, P., Wilson, T. L., et al. 1999, Earth, Moon and Planets, 78, 21

Biver, N., Bockelée-Morvan, D., Colom, P., et al. 1997, Science, 275, 1915

Biver, N., Bockelée-Morvan, D., Colom, P., et al. 1999, Earth, Moon and Planets, 78, 5

Bockelée-Morvan, D., \& Crovisier, J. 1985, A\&A, 151, 90

Bockelée-Morvan, D., Lis, D. C., Wink, J. E., et al. 2000, A\&A, 353, 1101

Boehnhardt, H., Birkle, K., Fiedler, A., et al. 1999, Earth, Moon and Planets, 78, 179

Bus, S. J., A’Hearn, M. F., Schleicher, D. G., \& Bowell, E. 1991, Science, 251, 774

Cochran, A. L. 1985, AJ, 90, 2609

Cochran, A. L., \& Cochran, W. D. 1991, Icarus, 90, 172

Cochran, A. L., Barker, E. S., Ramseyer, T. F., \& Storrs, A. D. 1992, Icarus, 98,151
Combi, M. R., \& Delsemme, A. H. 1980, ApJ, 237, 633

Combi, M. R., Kabin, K., DeZeeuw, D. L., \& Gombosi, T. I. 1999, Earth, Moon and Planets, 78, 275

Crovisier, J., \& Schloerb, F. P. 1991, in Comets in the Post-Halley era, ed. R. L. Newburn, M. Neugebauer, \& J. Rahe (Kluwer Academice Publisher), vol. 1, 149

Crovisier, J. 1994, JGR, 99, 3777

Dello Russo, N., Mumma, M. J., DiSanti, M. A., Magee-Sauer, K., \& Novak, R. 2001, Icarus, 153, 162

Enzian, A., Cabot, H., \& Klinger, J. 1997, A\&A, 319, 995

Fink, U., Combi, M. R., \& DiSanti, M. A. 1991, ApJ, 383, 356

Fitzsimmons, A., \& Cartwright, I. M. 1996, MNRAS, 278, L37

Glinski, R. J., Post, E. A., \& Anderson, C. M. 2001, ApJ, 550, 1131

Haser, L. 1957, Bulletin de la Société Royale des Sciences de Liège, 43, 740

Huebner, W. F., Keady, J. J., \& Lyon, S. P. 1992, Ap\&SS, 195, 1

Jackson, W. M. 1976, J. Photochem, 5, 107

Kawakita, H., \& Watanabe, J. 2002, ApJ, 572, L177

Kawakita, H., Watanabe, J., Kinoshita, D., et al. 2001a, PASJ, 53, L5

Kawakita, H., Watanabe, J.-I., Ando, H., et al. 2001b, Science, 294, 1089

Kurucz, R. L., Furenlid, I., Brault, J., \& Testerman, L. 1984, Solar flux atlas from 296 to $1300 \mathrm{~nm}$ (Sunspot, New Mexico: National Solar Observatory)

Meijere, A., Faber, D., Heinecke, U., et al. 2001, European J. Organic Chem., 663

Mumma, M. J., Disanti, M. A., dello Russo, N., et al. 1996, Science, 272,1310

Newburn, R. L., \& Spinrad, H. 1989, AJ, 97, 552

Rauer, H., Arpigny, C., Boehnhardt, H., et al. 1997, Science, 275, 1909

Schleicher, D. G. 1983, Ph.D. Thesis, University of Maryland

Schleicher, D. G., Lederer, S. M., Millis, R. L., \& Farnham, T. L. 1997, Science, 275, 1913

Senay, M. C., \& Jewitt, D. 1994, Nature, 371, 229

Senay, M. C., \& Jewitt, D. 1996, IAUC, 6312

Stief, L.-J., Donn, B., Glicker, S., Gentieu, E.-P., \& Mentall, J.-E. 1972, ApJ, 171, 21

Weaver, H. A., Brooke, T. Y., Chin, G., et al. 1999, Earth, Moon and Planets, 78, 71

Womack, M., Lutz, B. L., \& Wagner, R. M. 1994, ApJ, 433, 886

Wyckoff, S., Tegler, S., Wehinger, P. A., Spinrad, H., \& Belton, M. J. S. 1988, ApJ, 325, 927

Yamamoto, T. 1981, The Moon and Planets, 24, 453 